\title{
Review
}

\section{Heart Rate Variability and Pain: A Systematic Review}

\author{
Giuseppe Forte ${ }^{1,2, *(\mathbb{D}}$, Giovanna Troisi ${ }^{3}$, Mariella Pazzaglia ${ }^{1,2}$ (D), Vilfredo De Pascalis ${ }^{1}$ (D) \\ and Maria Casagrande $3, *$ (D)
}

1 Department of Psychology, "Sapienza" University of Rome, 00185 Rome, Italy; mariella.pazzaglia@uniroma1.it (M.P.); vilfredo.depascalis@uniroma1.it (V.D.P.)

2 Body and Action Lab, IRCCS Fondazione Santa Lucia, Via Ardeatina 306, 00179 Rome, Italy

3 Department of Clinical and Dynamic Psychology and Health Studies, "Sapienza" University of Rome, 00185 Rome, Italy; troisi.1862006@studenti.uniroma1.it

* Correspondence: g.forte@uniroma1.it (G.F.); maria.casagrande@uniroma1.it (M.C.)

Citation: Forte, G.; Troisi, G.; Pazzaglia, M.; Pascalis, V.D.; Casagrande, M. Heart Rate Variability and Pain: A Systematic Review. Brain Sci. 2022, 12, 153. https://doi.org/10.3390/ brainsci12020153

Academic Editor: Agorastos Agorastos

Received: 27 December 2021

Accepted: 21 January 2022

Published: 24 January 2022

Publisher's Note: MDPI stays neutral with regard to jurisdictional claims in published maps and institutional affiliations.

Copyright: (c) 2022 by the authors. Licensee MDPI, Basel, Switzerland. This article is an open access article distributed under the terms and conditions of the Creative Commons Attribution (CC BY) license (https:// creativecommons.org/licenses/by/ $4.0 /)$.

\begin{abstract}
Background and Objective: Heart rate variability (HRV) as an index of the autonomic nervous system appears to be related to reactivity to experimental pain stimuli. HRV could better explain the contributions of sympathetic and parasympathetic activity response to nociceptive stimulation. The aim of this study was to systematically review and synthesize the current evidence on HRV in relation to the experience of pain in experimental tasks. Databases and Data Treatment: Studies indexed in the PubMed, PsycINFO, MEDLINE, WebOfScience, and Scopus databases were reviewed for eligibility. Studies on the autonomic response (i.e., HRV) to experimentally induced pain in healthy adults were included. Different methods of pain induction were considered (e.g., thermal, pressure, and electrical). Data were synthesized considering the association between HRV and both pain induction and subjective measures of pain. Results: Seventy-one studies were included. The results underline significant change in both the sympathetic and parasympathetic autonomic nervous systems during the painful stimulation independent of the pain induction method. The autonomic reaction to pain could be affected by several factors, such as sex, age, body mass index, breathing patterns, the intensity of the stimulation, and the affective state. Moreover, an association between the autonomic nervous system and the subjective experience of pain was found. Higher parasympathetic activity was associated with better self-regulation capacities and, accordingly, a higher pain inhibition capacity. Conclusions: HRV appears to be a helpful marker to evaluate nociceptive response in experimentally induced pain. Future studies are also needed in clinical samples to understand better the interindividual changes of autonomic response due to pain stimuli.
\end{abstract}

Keywords: pain; heart rate variability; autonomic response

\section{Introduction}

Pain is defined as an aversive sensory and emotional experience typically caused by (or resembling that caused by) actual or potential tissue injury [1,2]. Accordingly, it is highlighted that (1) pain is always a subjective experience influenced by biological, psychological, and social factors (differently from nociception) and should be accepted and respected as such, (2) individuals learn the concept of pain through their life experiences, (3) there are several behaviors to communicate it aside from verbal description, and (4) it has an adaptive role, but it can have adverse effects on the individual's well-being [1].

Depending on the duration, pain can be acute or chronic [3]. While acute pain is considered an adaptive signal that prevents danger and guarantees survival [4], chronic (or persistent) pain is defined as having persisted for at least 3 months [4], and it usually matches chronic diseases and non-treated medical pathologies, affecting the individual's quality of life [5].

Furthermore, pain can be defined as "somatic" when it involves the skin, subcutaneous tissues, bones, muscles, blood vessels, or connective tissues or "visceral" when it affects the 
internal organs or the linings of the body cavities [5]. The former can arise from thermal, mechanical, and chemical stimuli, while the latter results from distension or prolonged contraction of the smooth muscle wall of the structure [6]. Both types can be elicited in experimental settings (e.g., [7,8]). Currently, self-reporting measures are the most employed method for assessing experimentally induced pain [9]. For example, pain intensity can be measured by adopting visual analogue scales (VASs), numerical rating scales (NRSs) [10], and verbal rating scales (VRSs) [11].

In acute pain, somatic and visceral noxious stimuli excite the nociceptors [12] and are converted into nerve impulses in order to allow the brain to read them and produce the conscious pain sensation [5]. However, nociception is only a component of the pain experience. In fact, pain sensitivity can be affected by many factors, such as mood, affective regulation strategies, and mental disorders [13]. Pain is a stressor, and it has been considered a specific emotion that reflects homeostatic behavioral drive $[13,14]$. The affective-motivational and the cognitive-evaluative components enhance the individual's organization of emotional and behavioral responses [5].

A comprehensive framework to investigate how organisms respond and adapt themselves to diverse types of stressors, including pain, is the Neurovisceral Integration Model (NVIM) [15-17]. The authors of the NVIM proposed a core set of neural structures, referred to as the Central Autonomic Network (CAN), which provides the ability to continuously assess and prepare the organism for an appropriate response [18]. In the NVIM, heart rate variability (HRV) has been proposed as an index of flexible and adaptive regulation of the nervous system to organize a homeostatic response to environmental requests and which is related to cognitive functions [18-22]. HRV represents the change in the time interval between successive heartbeats. It is considered a sensitive, non-invasive measurement of autonomic input to the heart [23]. It might provide measures of autonomic nervous system activity in both sympathetic (SNS) and parasympathetic (PNS) branches [23]. Since systems involved in autonomic control (such as periaqueductal gray, the insular and anterior cingulate cortices, amygdala, prefrontal cortex, and nucleus of the solitary tract) [24] are strictly connected with those involved in pain perception, HRV can be considered a reliable index of ANS reactivity to nociceptive stimulation [24-26].

Furthermore, HRV appears to be an index of baroreflex activity [15-17], one of the body's homeostatic mechanisms that maintains the blood pressure at a constant level. Patients with chronic pain show a reduction in HRV and baroreflex sensibility due to changes in efferent sympathetic and parasympathetic cardiac activity, which shift the balance to a sympathetic tone prevalence related to catecholamine release $[27,28]$.

$\mathrm{HRV}$ is also related to endogenous pain modulation (EPM), a relevant factor in chronic pain development and maintenance [29]. In fact, EPM depends on the excitation-inhibition balance, and HRV can be used as an index of the inhibitory processes involved in these neurovisceral networks. Furthermore, EPM and HRV are connected in both the presence and absence of chronic pain. Accordingly, HRV has several advantages in studies investigating the physiological response to nociceptive stimulation [15-17].

Several studies focused on pain sensitivity adopted HRV as a measure of autonomic responses. A systematic review on this topic was conducted by Koenig et al. in 2014 [14]. The authors identified 20 studies showing an increase in sympathetic baroreflex activity and a decrease in vagal parasympathetic activity, as reflected by changes in the frequency domain measures of HRV. However, there has been an increasing number of studies about this relationship in recent years. Therefore, analyzing the new studies can be relevant. This paper aims to systematically summarize the achieved results on the relationship between pain and HRV.

\section{Method}

The review process was conducted according to the PRISMA Statement [30,31] to systematically analyze studies on the relationships between HRV and pain within healthy adult samples. The protocol has not been registered. 


\section{Research Strategies}

A systematic review was conducted by searching articles published in peer-reviewed journals using the PubMed, PsycINFO, MEDLINE, WebOfScience, and Scopus databases. The last research was conducted on 5 January 2021.

The search was restricted to publications published since 1996 (i.e., years of publication of the first guidelines on the standards of measurements, physiological interpretation, and clinical use of HRV (Task Force of the European Society of Cardiology and the North American Society of Pacing and Electrophysiology, hereafter referred to as Task Force, 1996). Articles focused on analyzing the association between pain and HRV were considered for inclusion. The search strategy used the following keywords: "pain"; "pain sensitivity"; "Heart Rate Variability"; "HRV"; and "IBI". The reference list of all included studies was screened for additional study citations.

Eligibility Criteria

The list of potential articles produced by systematic research was screened for eligibility. Studies that included one or more methods of experimental induction of pain and the measurement of HRV were selected. Studies that adopted at least one measure of subjective pain perception (e.g., pain thresholds) were judged as eligible. Studies that included participants with medical conditions which could potentially influence this relationship were excluded (e.g., chronic pain disorder, hypertension, and cancer survivors).

Study Selection

The initial search identified 6559 results imported to the Mendeley database. The screening was performed in two phases. After removing duplicates, the initial eligibility assessment was based on titles and abstracts. Two authors (G.T. and G.F.) independently examined the full texts to confirm the suitability of the studies for the following qualitative synthesis. Then, the full texts that fit the inclusion criteria were screened for the eligibility criteria. Finally, 71 studies were included in the review. During the whole process, disagreements were resolved by consulting a supervisor (M.C.). The selection processes are reported in Figure 1.

Data Collection and Quality Assessment

According to the PICOS approach [30], the following information was extracted from each selected study: (1) author(s) and year of publication, (2) country, (3) sample size and female and male distribution, (4) age of participants, (5) method of pain induction, (6) pain assessment, (7) the main focus of the study, (8) derived HRV measures, and (9) findings. The data are reported in Table 1. 


\section{Identification of studies via databases and registers}

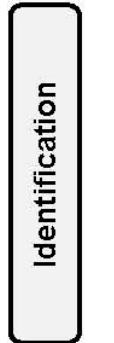

Records identified from:

Databases $(n=6559)$

- Psychlnfo: 504

- MEDLINE: 1551

- PubMed: 2629

- Scopus: 201

- Web of Science: 1674

Other sources $(n=3)$

Records removed before screening:

Records removed after filters $(n=2440)$

Duplicate records removed

$(n=1264)$
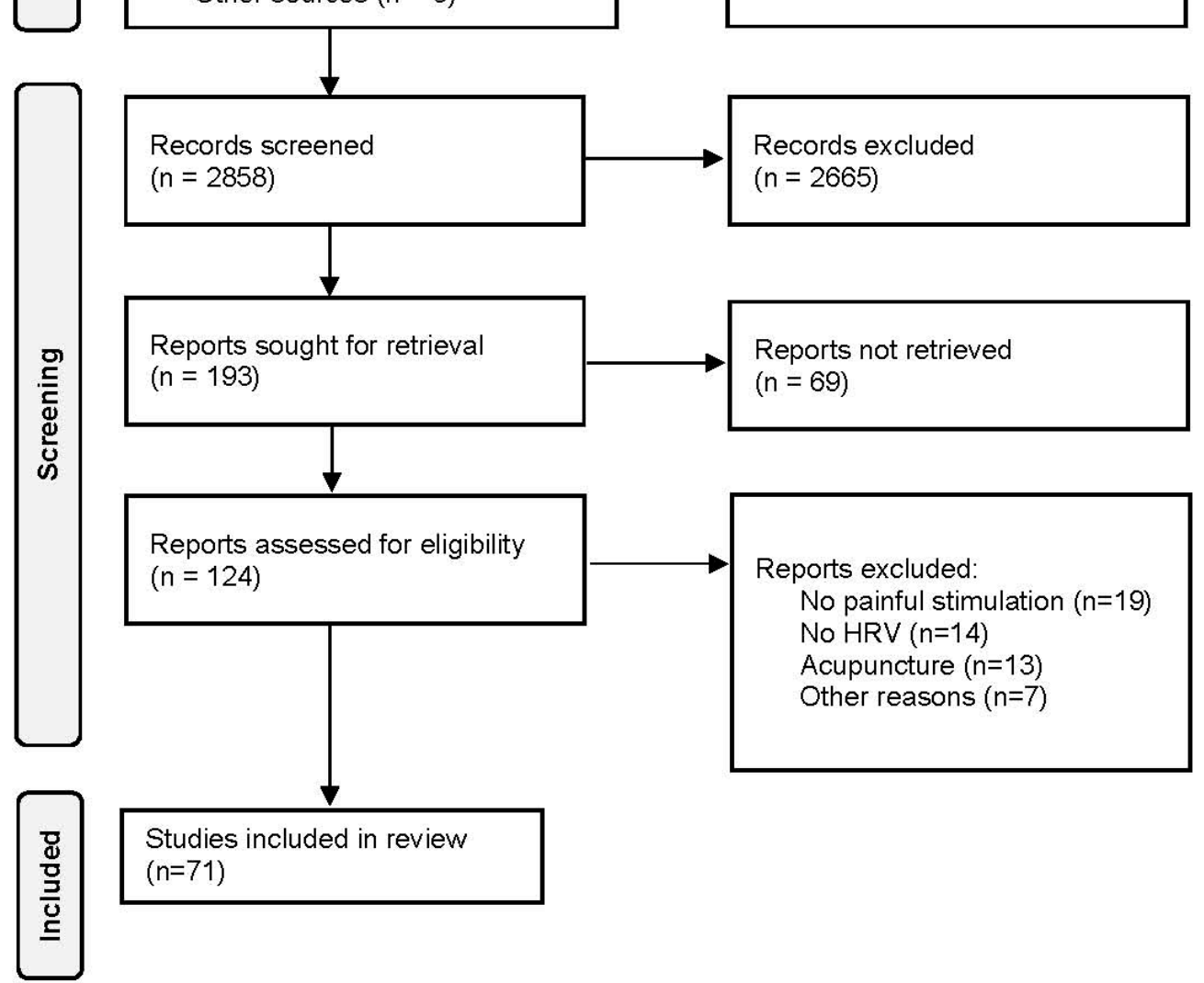

Studies included in review $(n=71)$

Figure 1. PRISMA flow diagram. 
Table 1. Characteristics of selected studies.

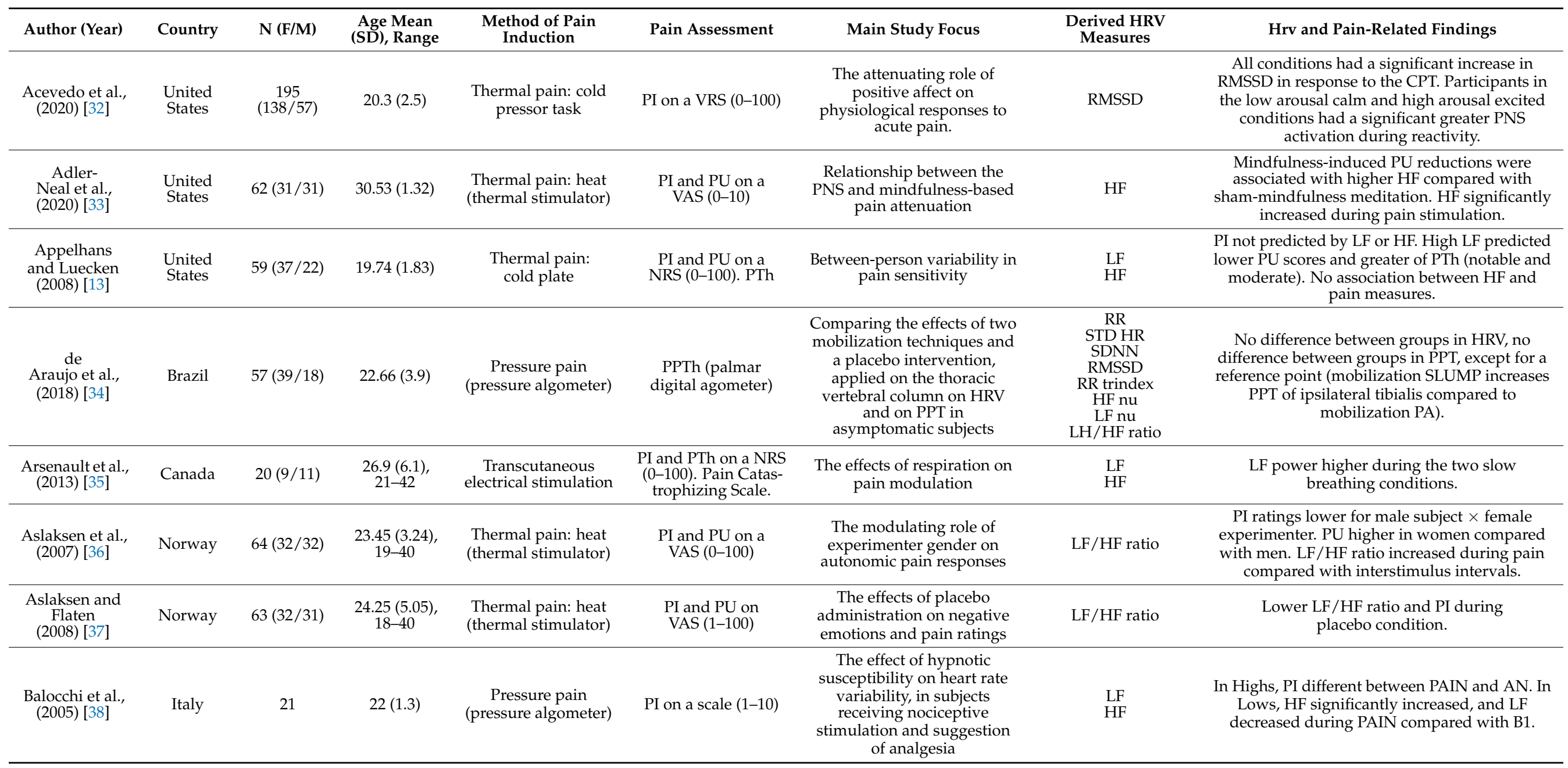


Table 1. Cont.

\begin{tabular}{|c|c|c|c|c|c|c|c|c|}
\hline $\begin{array}{l}\text { Author } \\
\text { (Year) }\end{array}$ & Country & $\mathbf{N}(\mathbf{F} / \mathbf{M})$ & $\begin{array}{c}\text { Age Mean } \\
\text { (SD), } \\
\text { Range }\end{array}$ & $\begin{array}{l}\text { Method of Pain } \\
\text { Induction }\end{array}$ & Pain Assessment & Main Study Focus & $\begin{array}{l}\text { Derived HRV } \\
\text { Measures }\end{array}$ & Hrv and Pain-Related Findings \\
\hline $\begin{array}{l}\text { Bendixen et al., } \\
\text { (2012) [39] }\end{array}$ & Denmark & $16(16 / 0)$ & $22.9(2.4)$ & $\begin{array}{l}\text { Muscle pain: } \\
\text { injection of } \\
\text { hypertonic saline } \\
\text { solution; } \\
\text { thermal pain: cold } \\
\text { pressor test }\end{array}$ & $\begin{array}{l}\text { PI and PU on a } \\
\text { NRS }(0-10) \text {, pain } \\
\text { on palpation } \\
\text { (POP) on a } \\
\text { NRS }(0-100)\end{array}$ & $\begin{array}{l}\text { The modulating role of } \\
\text { CPT and PASAT on } \\
\text { muscle pain and } \\
\text { autonomic function }\end{array}$ & $\begin{array}{l}\text { Mean RR } \\
\text { RMSSD } \\
\text { SDNN } \\
\text { LF } \\
\text { HF }\end{array}$ & $\begin{array}{l}\text { Decreased RMSSD, HF, and CCV-HF } \\
\text { during CPT. PI and PU higher in HS1 than } \\
\text { HS2 during CPT and PASAT conditions. }\end{array}$ \\
\hline $\begin{array}{l}\text { Boggero and } \\
\text { Segerstrom } \\
(2019 a)[41]\end{array}$ & $\begin{array}{l}\text { United } \\
\text { States }\end{array}$ & $\begin{array}{c}100 \\
(62 / 38)\end{array}$ & $\begin{array}{c}\text { Younger } \\
\text { adults: } \\
\text { 19.06 (1.81), } \\
18-28 ; \\
\text { older } \\
\text { adults: } \\
73.44(4.73), \\
65-84 \\
\end{array}$ & $\begin{array}{l}\text { Thermal pain: } \\
\text { hand immersion } \\
\text { in cold water }\end{array}$ & PI on a VRS $(0-10)$ & $\begin{array}{l}\text { Strategies employed by } \\
\text { younger and older adults } \\
\text { in order to maintain the } \\
\text { affective well-being after } \\
\text { an acute pain }\end{array}$ & Log HRV & $\begin{array}{c}\text { Older adults demonstrated significantly } \\
\text { lower HRV than younger adults. No } \\
\text { correlations between pain and HRV } \\
\text { were reported. }\end{array}$ \\
\hline $\begin{array}{l}\text { Boggero and } \\
\text { Segerstrom } \\
(2019 b)[42]\end{array}$ & $\begin{array}{l}\text { United } \\
\text { States }\end{array}$ & $\begin{array}{c}240 \\
(122 / 118)\end{array}$ & $\begin{array}{c}19.38(2.39) \\
18-39\end{array}$ & $\begin{array}{l}\text { Pressure pain } \\
\text { (pressure } \\
\text { algometer); } \\
\text { thermal: } \\
\text { immersion of the } \\
\text { non-dominant foot } \\
\text { in cold water }\end{array}$ & PPTh & $\begin{array}{l}\text { The relationship between } \\
\text { self-regulatory ability and } \\
\text { the experience of pain }\end{array}$ & $\log H F$ & $\begin{array}{c}\text { No relationship between pain and HRV } \\
\text { was found. }\end{array}$ \\
\hline $\begin{array}{l}\text { Bourassa et al., } \\
\text { (2019) [43] }\end{array}$ & $\begin{array}{l}\text { United } \\
\text { States }\end{array}$ & $\begin{array}{c}102 \\
(77 / 25)\end{array}$ & $19.1(1.75)$ & $\begin{array}{l}\text { Thermal pain: } \\
\text { cold pressor task }\end{array}$ & PI on NRS (0-10) & $\begin{array}{l}\text { The mediating role of a } \\
\text { romantic partner in } \\
\text { cardiovascular responses } \\
\text { during the cold } \\
\text { pressor task }\end{array}$ & RSA & $\begin{array}{l}\text { PI significantly lower in the partner } \\
\text { present condition compared with control } \\
\text { and mental activation conditions. No } \\
\text { significant differences in HRV } \\
\text { between conditions. }\end{array}$ \\
\hline
\end{tabular}


Table 1. Cont.

\begin{tabular}{|c|c|c|c|c|c|c|c|c|}
\hline $\begin{array}{l}\text { Author } \\
\text { (Year) }\end{array}$ & Country & $\mathbf{N}(\mathbf{F} / \mathrm{M})$ & $\begin{array}{l}\text { Age Mean } \\
\text { (SD), } \\
\text { Range }\end{array}$ & $\begin{array}{l}\text { Method of Pain } \\
\text { Induction }\end{array}$ & Pain Assessment & Main Study Focus & $\begin{array}{l}\text { Derived HRV } \\
\text { Measures }\end{array}$ & Hrv and Pain-Related Findings \\
\hline $\begin{array}{l}\text { Burton et al., } \\
\text { (2009) [44] }\end{array}$ & Australia & $26(13 / 13)$ & 28 & $\begin{array}{c}\text { Muscle and } \\
\text { subdermal pain: } \\
\text { injection of sterile } \\
\text { hypertonic saline } \\
\text { solution }\end{array}$ & PI on a VAS $(0-10)$ & $\begin{array}{l}\text { The effects of deep and } \\
\text { superficial pain on } \\
\text { muscle sympathetic } \\
\text { nerve activity }\end{array}$ & $\begin{array}{c}\text { LF } \\
\text { HF } \\
\text { LF } / \text { HF ratio }\end{array}$ & $\begin{array}{l}\text { Significant increase in LF/HF ratio during } \\
\text { both muscle and superficial pain. }\end{array}$ \\
\hline $\begin{array}{l}\text { Chalaye et al., } \\
\text { (2009) [45] }\end{array}$ & Canada & $20(9 / 11)$ & $25.1(5.6)$ & $\begin{array}{l}\text { Thermal pain: } \\
\text { heat (thermal } \\
\text { stimulator) }\end{array}$ & PTh; PTo. & $\begin{array}{l}\text { The effects of breathing } \\
\text { on heat pain } \\
\text { and autonomic } \\
\text { cardiac activity }\end{array}$ & $\begin{array}{l}\text { SDNN } \\
\text { LF power } \\
\text { HF power }\end{array}$ & $\begin{array}{l}\text { SDNN and LF power significantly } \\
\text { increased during pain in deep breathing } \\
\text { and HR Biofeedback conditions. No } \\
\text { significant differences in HF power. } \\
\text { PTh significantly higher during slow deep } \\
\text { breathing, HR Biofeedback and distraction } \\
\text { conditions; PTo higher in slow deep } \\
\text { breathing and HR Biofeedback conditions. }\end{array}$ \\
\hline $\begin{array}{c}\text { Cho } \\
\text { (2019) [46] }\end{array}$ & Korea & $45(21 / 24)$ & $22.4(1.49)$ & Electrical stimuli & PTh & $\begin{array}{l}\text { The effects of electrical } \\
\text { stimulation on the } \\
\text { autonomic } \\
\text { nervous system }\end{array}$ & HRV & $\begin{array}{l}\text { HRV significantly different between the } \\
\text { HF-Li and LF-Hi groups immediately after } \\
\text { stimulation and between the HF-LI and } \\
\text { LF-Hi groups } 30 \text { min after stimulation. }\end{array}$ \\
\hline $\begin{array}{l}\text { Chouchou et al., } \\
\text { (2011) [47] }\end{array}$ & France & $14(4 / 10)$ & $32.8(7.3)$ & $\begin{array}{l}\text { Thermal pain: } \\
\text { heat (laser) }\end{array}$ & $\begin{array}{l}\text { PTh on a } \\
\text { Likert-type scale } \\
\quad(0-10)\end{array}$ & $\begin{array}{l}\text { The assessment of } \\
\text { autonomic responses to } \\
\text { pain during sleep }\end{array}$ & $\begin{array}{c}\text { Mean RR } \\
\text { Wavelet power } \\
\text { coefficient of } \\
\text { LF, } \\
\text { HF, and } \\
\text { LF/HF ratio }\end{array}$ & $\begin{array}{l}\text { RR significantly decreased after the stimuli. } \\
\text { LF and LF/HF ratio significantly increased } \\
\text { after the stimuli. } \\
\text { No significant differences in HF. }\end{array}$ \\
\hline $\begin{array}{l}\text { Cotton et al., } \\
(2018)[48]\end{array}$ & $\begin{array}{l}\text { United } \\
\text { States }\end{array}$ & $34(26 / 8)$ & $\begin{array}{c}43.18 \\
(11.68)\end{array}$ & $\begin{array}{l}\text { Thermal pain: } \\
\text { heat (thermal } \\
\text { stimulator) }\end{array}$ & $\begin{array}{c}\text { PI on a VAS } \\
(0-200) \text { PU on a } \\
\text { VAS }(-100-100)\end{array}$ & $\begin{array}{l}\text { Autonomic responses to } \\
\text { pain in yoga practitioners } \\
\text { compared to a } \\
\text { control group }\end{array}$ & $\begin{array}{l}\text { RSA } \\
\text { SDRR } \\
\text { RMSSD } \\
\text { pNN50 }\end{array}$ & $\begin{array}{c}\text { Yogis had significantly slower RSA during } \\
\text { baseline compared with controls. } \\
\text { Controls had lower RSA during pain than } \\
\text { during warm trials. Yogis had the same } \\
\text { lever of RSA during both pain and } \\
\text { warm trials. }\end{array}$ \\
\hline
\end{tabular}


Table 1. Cont.

\begin{tabular}{|c|c|c|c|c|c|c|c|c|}
\hline $\begin{array}{l}\text { Author } \\
\text { (Year) }\end{array}$ & Country & $\mathbf{N}(\mathbf{F} / \mathbf{M})$ & $\begin{array}{l}\text { Age Mean } \\
\text { (SD), } \\
\text { Range }\end{array}$ & $\begin{array}{c}\text { Method of } \\
\text { Pain Induction }\end{array}$ & Pain Assessment & Main Study Focus & $\begin{array}{l}\text { Derived HRV } \\
\text { Measures }\end{array}$ & Hrv and Pain-Related Findings \\
\hline $\begin{array}{l}\text { Courtois et al., } \\
\text { (2020) [49] }\end{array}$ & Belgium & $\begin{array}{l}\text { Ex 1: } \\
31(31 / 0) \\
\text { Ex 2: } 28 \\
(28 / 0) \\
\text { Ex 3: } 24 \\
(24 / 0)\end{array}$ & $\begin{array}{l}\text { Ex 1: } 22.45 \\
\quad(3.10) \\
\text { Ex 2: } 20.25 \\
\quad(2.50) ; \\
\text { Ex 3: } 22.55 \\
\quad(3.16)\end{array}$ & $\begin{array}{l}\text { Ex 1: electrical } \\
\text { pain; } \\
\text { Ex 2: Thermal } \\
\text { (thermal } \\
\text { stmulator); } \\
\text { Ex 3: Mechanical } \\
\text { pain (pressure } \\
\text { algometer) }\end{array}$ & $\begin{array}{l}\text { PTh. PI on a } \\
\text { NRS (0-10) }\end{array}$ & $\begin{array}{l}\text { The effect of slow deep } \\
\text { breathing (SDB) on pain } \\
\text { sensitivity, HRV, and } \\
\text { baroreflex sensitivity }\end{array}$ & RMSSD & $\begin{array}{l}\text { RMSSD increased during SDB in all } \\
\text { experimental conditions. No differences in } \\
\text { pain ratings were found, nor in } \\
\text { relationships between subjective pain } \\
\text { and HRV. }\end{array}$ \\
\hline $\begin{array}{l}\text { De Pascalis } \\
\text { and Scacchia } \\
(2019)[50]\end{array}$ & Italy & $65(65 / 0)$ & $\begin{array}{c}24.5(2.5) \\
18-36\end{array}$ & $\begin{array}{l}\text { Thermal pain: } \\
\text { cold cup test }\end{array}$ & $\begin{array}{l}\text { Pain expectation } \\
\text { and PI on a } \\
\text { NRS }(0-100)\end{array}$ & $\begin{array}{l}\text { The influence of } \\
\text { personality traits on } \\
\text { placebo analgesia }\end{array}$ & $\begin{array}{c}\text { RR } \\
\text { SDNN } \\
\text { LF power } \\
\text { HF power } \\
\text { LF/HF ratio }\end{array}$ & $\begin{array}{l}\text { Negative correlation was found between } \\
\text { pain and time domain but not between } \\
\text { pain and frequency domain. }\end{array}$ \\
\hline $\begin{array}{l}\text { Dodo and } \\
\text { Hashimoto } \\
(2017)[51]\end{array}$ & Japan & 74 & $21.14(2.93)$ & $\begin{array}{l}\text { Thermal pain: } \\
\text { cold pressor test }\end{array}$ & $\begin{array}{l}\text { Pain perception on } \\
\text { the Wong-Baker } \\
\text { Faces Pain Rating } \\
\text { Scale, PI on a } \\
\text { scale }(0-5)\end{array}$ & $\begin{array}{l}\text { The relationship between } \\
\text { anxiety sensitivity and } \\
\text { autonomic responses } \\
\text { during pain }\end{array}$ & $\begin{array}{l}\text { CVI } \\
\text { CSI }\end{array}$ & $\begin{array}{l}\text { CVI: significantly higher during CPT in } \\
\text { both the low-AS and the high-AS group; } \\
\text { low group also higher in recovery } \\
\text { compared with rest; during recovery, } \\
\text { significantly higher in low-As group than } \\
\text { in the high-As group. Subjective pain } \\
\text { higher in high-As group than low-As } \\
\text { group post-CPT. }\end{array}$ \\
\hline $\begin{array}{l}\text { Evans et al., } \\
\text { (2014) [52] }\end{array}$ & $\begin{array}{l}\text { United } \\
\text { States }\end{array}$ & $63(29 / 34)$ & $18.98(1.62)$ & $\begin{array}{l}\text { Thermal pain: } \\
\text { cold pressor task }\end{array}$ & $\begin{array}{l}\text { PTo (total time } \\
\quad \text { in sec) }\end{array}$ & $\begin{array}{l}\text { The effects of brief } \\
\text { mindfulness instructions } \\
\text { on pain tolerance } \\
\text { and HRV }\end{array}$ & Log HF power & $\begin{array}{l}\text { Higher HRV at baseline positively } \\
\text { correlated with greater PTo in the } \\
\text { control group. }\end{array}$ \\
\hline $\begin{array}{l}\text { Fauchon et al., } \\
\text { (2017) [53] }\end{array}$ & France & $40(20 / 20)$ & $23.2(8.2)$ & $\begin{array}{l}\text { Thermal pain: } \\
\text { heat (thermal } \\
\text { stimulator) }\end{array}$ & $\begin{array}{l}\text { PI on a VAS } \\
(0-100) ; \text { PTh }\end{array}$ & $\begin{array}{l}\text { The effect of perceived } \\
\text { support on pain } \\
\text { modulation and } \\
\text { associated vegetative } \\
\text { reactions }\end{array}$ & IBI & No correlation between IBI and pain. \\
\hline
\end{tabular}


Table 1. Cont.

\begin{tabular}{|c|c|c|c|c|c|c|c|c|}
\hline $\begin{array}{l}\text { Author } \\
\text { (Year) }\end{array}$ & Country & $\mathrm{N}(\mathrm{F} / \mathrm{M})$ & $\begin{array}{l}\text { Age Mean } \\
\text { (SD), } \\
\text { Range }\end{array}$ & $\begin{array}{l}\text { Method of Pain } \\
\text { Induction }\end{array}$ & Pain Assessment & Main Study Focus & $\begin{array}{l}\text { Derived HRV } \\
\text { Measures }\end{array}$ & Hrv and Pain-Related Findings \\
\hline $\begin{array}{l}\text { Fauchon et al., } \\
\text { (2018) [54] }\end{array}$ & France & $76(17 / 59)$ & $27.8(6.3)$ & $\begin{array}{l}\text { Thermal pain: } \\
\text { heat (thermal } \\
\text { stimulator) }\end{array}$ & n.r. & $\begin{array}{l}\text { The role of context in the } \\
\text { autonomic responses to } \\
\text { acute pain }\end{array}$ & $\begin{array}{c}\text { LF } \\
\text { HF } \\
\mathrm{LF} / \mathrm{HF} \text { ratio }\end{array}$ & $\begin{array}{l}\text { LF/HF ratio significantly increased in } \\
\text { response to pain only during } \\
\text { unempathetic condition. Higher LF during } \\
\text { unempathetic condition than in } \\
\text { neutral condition. }\end{array}$ \\
\hline $\begin{array}{l}\text { Fazalbhoy et al., } \\
\text { (2012) [55] }\end{array}$ & Australia & $12(1 / 11)$ & $18-48$ & $\begin{array}{l}\text { Muscle pain: } \\
\text { injection of a } \\
\text { hypertonic } \\
\text { solution }\end{array}$ & $\begin{array}{l}\text { PI on a VAS }(0-10) \\
\text { McGill Pain } \\
\text { Questionnaire }\end{array}$ & $\begin{array}{l}\text { The cardiovascular } \\
\text { responses to tonic pain }\end{array}$ & $\begin{array}{c}\mathrm{LF} \\
\mathrm{HF} \\
\mathrm{LF} / \mathrm{HF} \text { ratio }\end{array}$ & $\begin{array}{l}\text { Increasing MSNA group: significantly } \\
\text { higher LF power and LF/HF ratio, lower } \\
\text { HF power and RMSSD. }\end{array}$ \\
\hline $\begin{array}{l}\text { Fidanza et al., } \\
\text { (2017) [56] }\end{array}$ & Italy & $51(28 / 23)$ & $20-27$ & $\begin{array}{l}\text { Electrical } \\
\text { stimulation; } \\
\text { thermal pain: cold } \\
\text { pressor test }\end{array}$ & PI $(0-10)$ & $\begin{array}{l}\text { The relationship between } \\
\text { pain modulation } \\
\text { (suggestion of analgesia } \\
\text { VS Diffuse Noxious } \\
\text { Inhibitory Control) } \\
\text { and hypnotizability }\end{array}$ & $\mathrm{LF} / \mathrm{HF}$ ratio & $\begin{array}{l}\text { HRV was not modulated by } \\
\text { pain experience. }\end{array}$ \\
\hline $\begin{array}{l}\text { Geva et al., } \\
(2017)[58]\end{array}$ & Israel & $25(0 / 25)$ & $35.9(10)$ & $\begin{array}{l}\text { Thermal pain: } \\
\text { heat (thermal } \\
\text { stimulator) }\end{array}$ & $\begin{array}{l}\text { PTh and Pto with } \\
\text { the thermal } \\
\text { stimulator; PI on a } \\
\text { VAS }(0-10)\end{array}$ & $\begin{array}{l}\text { Loss of pain modulation } \\
\text { under acute psychosocial } \\
\text { stress in triathletes }\end{array}$ & HRV & $\begin{array}{l}\text { HRV correlated negatively with the } \\
\text { reduction in CPM due to stress. }\end{array}$ \\
\hline $\begin{array}{l}\text { Ghione et al., } \\
\text { (2004) [59] }\end{array}$ & Italy & $10(0 / 10)$ & $41(7)$ & $\begin{array}{l}\text { Electromagnetic } \\
\text { field exposure }\end{array}$ & PTh; PTo & $\begin{array}{l}\text { The effects of an } \\
\text { electromagnetic field on } \\
\text { pain perception and on } \\
\text { cardiovascular } \\
\text { parameters }\end{array}$ & $\begin{array}{l}\text { LF } \\
\text { HF }\end{array}$ & $\begin{array}{l}\text { HF progressively increased during sham } \\
\text { exposure and remained constant during } \\
\text { magnetic exposure. LF increased during } \\
\text { both types of exposure. }\end{array}$ \\
\hline
\end{tabular}


Table 1. Cont.

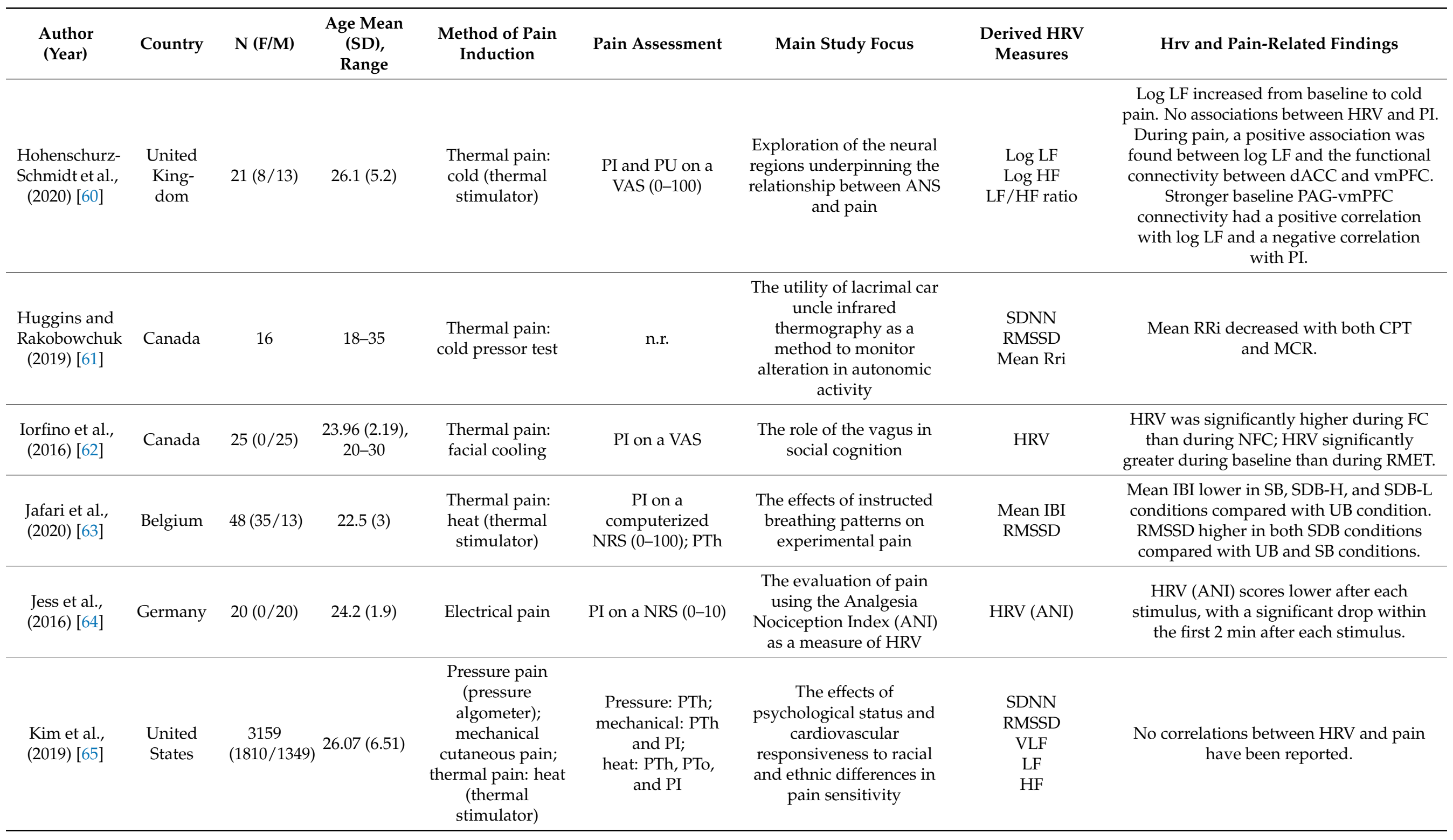


Table 1. Cont.

\begin{tabular}{|c|c|c|c|c|c|c|c|c|}
\hline $\begin{array}{l}\text { Author } \\
\text { (Year) }\end{array}$ & Country & $\mathbf{N}(\mathbf{F} / \mathbf{M})$ & $\begin{array}{l}\text { Age Mean } \\
\text { (SD), } \\
\text { Range }\end{array}$ & $\begin{array}{l}\text { Method of Pain } \\
\text { Induction }\end{array}$ & Pain Assessment & Main Study Focus & $\begin{array}{l}\text { Derived HRV } \\
\text { Measures }\end{array}$ & Hrv and Pain-Related Findings \\
\hline $\begin{array}{l}\text { Kobuch et al., } \\
\text { (2015) [66] }\end{array}$ & Australia & $50(25 / 25)$ & $\begin{array}{l}22.3(1.15) \\
18-39\end{array}$ & $\begin{array}{l}\text { Muscle pain: } \\
\text { injection of } \\
\text { hypertonic } \\
\text { saline solution }\end{array}$ & $\begin{array}{l}\text { PI on a linear } \\
\text { potentiometer } \\
\text { calibrated to the } \\
\text { NRS }(0-10) ; \\
\text { McGill Pain } \\
\text { Questionnaire }\end{array}$ & $\begin{array}{l}\text { The relationship between } \\
\text { baseline physiological } \\
\text { parameters and MSNA } \\
\text { responses to tonic } \\
\text { muscle pain }\end{array}$ & $\begin{array}{l}\text { LF } \\
\text { HF } \\
\text { LF/HF ratio } \\
\text { RMSSD }\end{array}$ & $\begin{array}{l}\text { No correlations between pain and HRV } \\
\text { have been found. }\end{array}$ \\
\hline $\begin{array}{l}\text { Luo et al., } \\
(2020)[68]\end{array}$ & China & $29(14 / 15)$ & $\begin{array}{l}19.93(1.6) \\
19-27\end{array}$ & $\begin{array}{l}\text { Thermal pain: } \\
\text { cold (holding a } \\
\text { bottle with } \\
\text { iced water) }\end{array}$ & PI on a scale (0-10) & $\begin{array}{l}\text { The role and mechanisms } \\
\text { of self-compassion in } \\
\text { pain perception }\end{array}$ & $\mathrm{HF}$ & $\begin{array}{l}\text { Increased HFs were associated with lower } \\
\text { PI in the self-compassion compared with } \\
\text { control condition. HF higher in } \\
\text { self-compassion compared with control. }\end{array}$ \\
\hline $\begin{array}{l}\text { Martin et al., } \\
(2012)[69]\end{array}$ & $\begin{array}{l}\text { United } \\
\text { States }\end{array}$ & $30(20 / 10)$ & $21(5.5)$ & $\begin{array}{l}\text { Electric } \\
\text { stimulation }\end{array}$ & $\begin{array}{l}\text { PI on a NRS } \\
(0-100) ; \text { PTh }\end{array}$ & $\begin{array}{l}\text { The influence of } \\
\text { experimentally } \\
\text { manipulated breathing } \\
\text { on pain }\end{array}$ & $\begin{array}{l}\text { Mean RR } \\
\text { RMSSD }\end{array}$ & $\begin{array}{l}\text { HRV changed during breathing } \\
\text { manipulation, but it was not correlated } \\
\text { with pain outcomes. }\end{array}$ \\
\hline $\begin{array}{l}\text { Matthewson et } \\
\text { (2019) [70] }\end{array}$ & $\begin{array}{l}t \text { alU, } \text { nited } \\
\text { States }\end{array}$ & $84(42 / 42)$ & $27.9(6.29)$ & $\begin{array}{l}\text { Thermal stimuli: } \\
\text { heat (thermal } \\
\text { stimulator) }\end{array}$ & $\begin{array}{l}\text { PI on a NRS } \\
\quad(0-100)\end{array}$ & $\begin{array}{l}\text { The role of cognitive } \\
\text { self-regulation in pain } \\
\text { experience and its effects } \\
\text { on autonomic responses }\end{array}$ & IBI & $\begin{array}{l}\text { Association was found between IBI } \\
\text { and pain. }\end{array}$ \\
\hline $\begin{array}{l}\text { Meeuse et al., } \\
(2013)[71]\end{array}$ & Netherlands & $73(44 / 29)$ & $30(11)$ & $\begin{array}{l}\text { Thermal pain: } \\
\text { heat (thermal } \\
\text { stimulator) }\end{array}$ & $\begin{array}{l}\text { PI on a VAS } \\
\quad(0-100)\end{array}$ & $\begin{array}{l}\text { The usefulness of HRV in } \\
\text { quantifying pain intensity }\end{array}$ & $\begin{array}{c}\text { IBI } \\
\operatorname{lnSDNN} \\
\operatorname{lnLF} \\
\operatorname{lnHF} \\
\text { LF/HF ratio }\end{array}$ & $\begin{array}{c}\text { lnSDNN and lnLF significantly decreased } \\
\text { during pain compared with baseline. No } \\
\text { significant correlation between PI and } \\
\text { HRV parameter was found. }\end{array}$ \\
\hline
\end{tabular}


Table 1. Cont.

\begin{tabular}{|c|c|c|c|c|c|c|c|c|}
\hline $\begin{array}{l}\text { Author } \\
\text { (Year) }\end{array}$ & Country & $\mathbf{N}(\mathrm{F} / \mathrm{M})$ & $\begin{array}{l}\text { Age Mean } \\
\text { (SD), } \\
\text { Range }\end{array}$ & $\begin{array}{l}\text { Method of Pain } \\
\text { Induction }\end{array}$ & Pain Assessment & Main Study Focus & $\begin{array}{l}\text { Derived HRV } \\
\text { Measures }\end{array}$ & Hrv and Pain-Related Findings \\
\hline $\begin{array}{l}\text { Nahman- } \\
\text { Averbuch et al., } \\
\text { (2016a) [72] }\end{array}$ & Israel & $40(20 / 20)$ & $26.45(3.85)$ & $\begin{array}{l}\text { Thermal pain: } \\
\text { heat (thermal } \\
\text { stimulator) and } \\
\text { cold (immersion of } \\
\text { a foot in } \\
\text { cold water); } \\
\text { mechanical pain }\end{array}$ & $\begin{array}{l}\text { PTh; PI on a NPS } \\
\quad(0-100)\end{array}$ & $\begin{array}{l}\text { Sex differences in the } \\
\text { relationship between pain } \\
\text { perception and HRV }\end{array}$ & $\begin{array}{c}\text { RMSSD } \\
\text { LF } \\
\text { HF } \\
\text { LF/HF ratio }\end{array}$ & $\begin{array}{l}\text { Women: LFnu significantly lower and } \\
\text { Hfnu significantly higher. } \\
\text { Men: higher RMSSD significantly } \\
\text { negatively correlated with higher pain } \\
\text { adaptation and with more efficient } \\
\text { CPM response. }\end{array}$ \\
\hline $\begin{array}{l}\text { Nahman- } \\
\text { Averbuch et al., } \\
(2016 b)[73]\end{array}$ & Israel & $40(20 / 20)$ & $26.45(3.85)$ & $\begin{array}{l}\text { Thermal pain: } \\
\text { heat (thermal } \\
\text { stimulator) and } \\
\text { cold (immersion of } \\
\text { a foot in } \\
\text { cold water); } \\
\text { mechanical pain }\end{array}$ & $\begin{array}{l}\text { M Pain: PI on a } \\
\text { NPS (0-100); PTh } \\
\text { CPM: PI of the TS } \\
\text { on a COVAS; CS } \\
\text { on an NPS }\end{array}$ & $\begin{array}{l}\text { The effects of oral } \\
\text { clonidine on } \\
\text { pain perception }\end{array}$ & $\begin{array}{l}\text { RMSSD } \\
\text { LF nu } \\
\text { HF nu } \\
\text { LF/HF ratio }\end{array}$ & $\begin{array}{l}\text { Higher RMSSD in clonidine group. No } \\
\text { differences found in the other } \\
\text { HRV parameters. }\end{array}$ \\
\hline $\begin{array}{l}\text { Nahman- } \\
\text { Averbuch et al., } \\
(2016 c)[74]\end{array}$ & Israel & $30(30 / 0)$ & $25.3(4.1)$ & $\begin{array}{l}\text { Thermal pain: } \\
\text { heat (thermal } \\
\text { stimulator and the } \\
\text { immersion of a } \\
\text { hand in hot water); } \\
\text { mechanical pain }\end{array}$ & $\begin{array}{l}\text { Thermal: PTh; PI } \\
\text { on a NPS (0-100); } \\
\text { mechanincal: PI } \\
\text { on a NPS (0-100) }\end{array}$ & $\begin{array}{l}\text { Effect of anxiety level on } \\
\text { parasympathetic function } \\
\text { and pain perception }\end{array}$ & $\begin{array}{l}\text { RMSSD } \\
\text { LF nu } \\
\text { HF nu } \\
\text { LF/HF ratio }\end{array}$ & $\begin{array}{l}\text { Increased parasympathetic activity during } \\
\text { recovery in both groups. In the } \\
\text { high-anxiety group, higher RMSSD during } \\
\text { baseline correlated with higher pain } \\
\text { ratings during tonic pain stimulus. }\end{array}$ \\
\hline $\begin{array}{c}\text { Olsson and } \\
\text { von Schéele } \\
(2011)[75]\end{array}$ & Sweden & $32(20 / 12)$ & $39.7(8.6)$ & Bed of nails & PI on a NRS $(0-10)$ & $\begin{array}{l}\text { Subjective physiologic } \\
\text { responses of lying on a } \\
\text { bed of nails (BN) }\end{array}$ & $\begin{array}{l}\text { SDNN log } \\
\text { LF log } \\
\text { HF log }\end{array}$ & $\begin{array}{l}\text { HF higher on the BN. Higher SDNN and } \\
\text { LF during relaxing instruction on CD } \\
\text { while lying on the BN. }\end{array}$ \\
\hline $\begin{array}{l}\text { Paine et al., } \\
\text { (2009a) [8] }\end{array}$ & $\begin{array}{l}\text { United } \\
\text { King- } \\
\text { dom }\end{array}$ & $19(11 / 8)$ & $22-54$ & $\begin{array}{l}\text { Visceral pain: } \\
\text { esophageal } \\
\text { balloon distension }\end{array}$ & PTo; PTh & $\begin{array}{l}\text { The relationship between } \\
\text { personality and } \\
\text { autonomic responses to } \\
\text { visceral pain }\end{array}$ & $\begin{array}{l}\text { CVC } \\
\text { CSI }\end{array}$ & $\begin{array}{l}\text { CSI increased during pain; no changes in } \\
\text { CVC during pain. }\end{array}$ \\
\hline $\begin{array}{l}\text { Paine et al., } \\
\text { (2009b) [76] }\end{array}$ & $\begin{array}{l}\text { United } \\
\text { King- } \\
\text { dom }\end{array}$ & $18(16 / 2)$ & $35.4(2.7)$ & $\begin{array}{l}\text { Visceral pain: } \\
\text { proximal and } \\
\text { distal balloon } \\
\text { distension; } \\
\text { somatic pain: } \\
\text { nail-bed } \\
\text { stimulation }\end{array}$ & $\begin{array}{l}\text { PI and PU on a } \\
\text { VRS (0-10); PTo } \\
\text { and PTh }\end{array}$ & $\begin{array}{l}\text { The relationship between } \\
\text { autonomic control and } \\
\text { personality in response to } \\
\text { visceral and somatic pain }\end{array}$ & $\begin{array}{l}\text { CVT } \\
\text { CSI }\end{array}$ & $\begin{array}{l}\text { Increased CVT in the } 90 \text { s post-stimulus } \\
\text { compared with pre-stimulus CVT. } \\
\text { Significantly greater increase in CVT for } \\
\text { distal balloon than for nail bed. }\end{array}$ \\
\hline
\end{tabular}


Table 1. Cont.

\begin{tabular}{|c|c|c|c|c|c|c|c|c|}
\hline $\begin{array}{l}\text { Author } \\
\text { (Year) }\end{array}$ & Country & $\mathbf{N}(\mathrm{F} / \mathrm{M})$ & $\begin{array}{l}\text { Age Mean } \\
\text { (SD), } \\
\text { Range }\end{array}$ & $\begin{array}{l}\text { Method of Pain } \\
\text { Induction }\end{array}$ & Pain Assessment & Main Study Focus & $\begin{array}{c}\text { Derived HRV } \\
\text { Measures }\end{array}$ & Hrv and Pain-Related Findings \\
\hline $\begin{array}{l}\text { Perlaki et al., } \\
\text { (2015) [77] }\end{array}$ & Hungary & $18(0 / 18)$ & $22.89(1.96)$ & $\begin{array}{l}\text { Thermal pain: } \\
\text { heat (thermal } \\
\text { stimulator) }\end{array}$ & $\begin{array}{c}\text { PI on a VAS }(0-10) ; \\
\text { PTh }\end{array}$ & $\begin{array}{l}\text { Investigating the brain } \\
\text { structures responsible for } \\
\text { pain-related } \\
\text { autonomic changes }\end{array}$ & $\begin{array}{c}\text { LF } \\
\text { HF } \\
\text { LF/HF ratio }\end{array}$ & $\begin{array}{c}\text { The median COPE of left MPFC showed } \\
\text { negative correlations with LF/HF ratio } \\
\text { and a positive correlation with HFnu. The } \\
\text { median COPE of right MPFC showed } \\
\text { significant negative correlations } \\
\text { with SDNN. }\end{array}$ \\
\hline $\begin{array}{l}\text { Picchiottino et } \\
(2020)[79]\end{array}$ & France & $41(22 / 19)$ & $19.9(3.5)$ & $\begin{array}{l}\text { Pressure pain } \\
\text { (pressure } \\
\text { algometer) }\end{array}$ & PTh & $\begin{array}{l}\text { The effect of spinal } \\
\text { manipulation on } \\
\text { cardiovascular autonomic } \\
\text { activity and the } \\
\text { relationship to pressure } \\
\text { pain threshold }\end{array}$ & $\begin{array}{c}\text { LF } \\
\text { HF } \\
\text { LF/HF ratio } \\
\text { RMSSD } \\
\text { SDNN }\end{array}$ & $\begin{array}{l}\text { Weak and moderate positive association } \\
\text { between changes in PTh and changes in } \\
\qquad \log \text { LF. }\end{array}$ \\
\hline $\begin{array}{l}\text { Piovesan et al., } \\
\text { (2019) [80] }\end{array}$ & $\begin{array}{l}\text { United } \\
\text { Kingdom }\end{array}$ & $40(30 / 10)$ & $26.2(3.91)$ & $\begin{array}{l}\text { Electrical pain; } \\
\text { thermal pain: heat } \\
\text { (thermal stimulator) }\end{array}$ & PI on a NRS $(0-10)$ & $\begin{array}{l}\text { The relationship between } \\
\text { autonomic nervous } \\
\text { system and perceived } \\
\text { duration of pain } \\
\text { experience }\end{array}$ & HF nu & $\begin{array}{l}\text { Only high-intensity stimuli were } \\
\text { associated with changes in HRV. } \\
\text { No relationship between heat pain and } \\
\text { HRV was found. }\end{array}$ \\
\hline $\begin{array}{l}\text { Pollatos et al., } \\
\text { (2012a) [81] }\end{array}$ & Germany (?) & $60(30 / 30)$ & $24.4(3.2)$ & $\begin{array}{l}\text { Pressure pain } \\
\text { (pressure algometer) }\end{array}$ & $\begin{array}{l}\text { PTh; PTo; PI and } \\
\text { PU on a VAS (1-9) }\end{array}$ & $\begin{array}{l}\text { The role of interception } \\
\text { sensitivity on cutaneous } \\
\text { pain perception }\end{array}$ & $\begin{array}{c}\text { LF } \\
\text { HF } \\
\text { LF/HF ratio }\end{array}$ & $\begin{array}{l}\text { HFnu significantly decreased while LFnu } \\
\text { and LF/HF ratio significantly increased } \\
\text { during pain. }\end{array}$ \\
\hline $\begin{array}{l}\text { Pollatos et al., } \\
\text { (2012b) [82] }\end{array}$ & Germany & $22(22 / 0)$ & $\begin{array}{c}24.4(2.8) \\
21-31\end{array}$ & $\begin{array}{c}\text { Pressure pain } \\
\text { (pressure algometer) }\end{array}$ & $\begin{array}{l}\text { PTh, PTo; PI and } \\
\text { PU on a scale (1-9) }\end{array}$ & $\begin{array}{l}\text { The effects of food } \\
\text { deprivation on pain } \\
\text { perception }\end{array}$ & $\begin{array}{c}\text { HF nu } \\
\text { LF/HF ratio }\end{array}$ & $\begin{array}{l}\text { Day 1: PTo positively correlated with HF } \\
\text { nu and inversely correlated with LF/HF } \\
\text { ratio. Experimental group: after } 24 \mathrm{~h} \text { of } \\
\text { food deprivation, significant positive } \\
\text { correlation between differences in HF and } \\
\text { PTh (hungry minus breakfast). }\end{array}$ \\
\hline
\end{tabular}


Table 1. Cont.

\begin{tabular}{|c|c|c|c|c|c|c|c|c|}
\hline $\begin{array}{l}\text { Author } \\
\text { (Year) }\end{array}$ & Country & $\mathbf{N}(\mathbf{F} / \mathbf{M})$ & $\begin{array}{l}\text { Age Mean } \\
\text { (SD), } \\
\text { Range }\end{array}$ & $\begin{array}{l}\text { Method of Pain } \\
\text { Induction }\end{array}$ & Pain Assessment & Main Study Focus & $\begin{array}{l}\text { Derived HRV } \\
\text { Measures }\end{array}$ & Hrv and Pain-Related Findings \\
\hline $\begin{array}{l}\text { Poulsen et al., } \\
\text { (2019) [83] }\end{array}$ & Denmark & $20(10 / 10)$ & $25.0(4.0)$ & $\begin{array}{c}\text { Capsaicin } \\
\text { application; } \\
\text { somatosensory } \\
\text { functions; thermal } \\
\text { pain: heat and } \\
\text { cold (thermal } \\
\text { stimulator); } \\
\text { mechanical pain } \\
\text { (calibrated von } \\
\text { Frey nylon } \\
\text { filaments) }\end{array}$ & $\begin{array}{l}\text { PI on a NRS } \\
(0-100)\end{array}$ & $\begin{array}{l}\text { The region-specific effects } \\
\text { of painful stimulation }\end{array}$ & $\begin{array}{l}\text { Mean RR } \\
\text { SDNN } \\
\text { RMSSD } \\
\text { LF } \\
\text { HF }\end{array}$ & $\begin{array}{l}\text { Higher mean RR, increased RMSSD, } \\
\text { SDNN, LF power, HF power, and CCV-HF } \\
\text { power during capsaicin stimulation. }\end{array}$ \\
\hline $\begin{array}{l}\text { Santarcangelo e } \\
\text { (2008) [84] }\end{array}$ & tal. Italy & $19(19 / 0)$ & 21 & $\begin{array}{l}\text { Pressure pain } \\
\text { (pressure } \\
\text { algometer) }\end{array}$ & PI on a scale $(0-10)$ & $\begin{array}{l}\text { Differences due to } \\
\text { hypnotizability in the } \\
\text { pain-related modulation } \\
\text { of HRV during suggestion } \\
\text { of analgesia }\end{array}$ & $\begin{array}{c}\text { Mean RR } \\
\text { HF } \\
\text { LF } \\
\text { LF/HF } \\
\text { SDNN } \\
\text { RMSSD } \\
\text { CSI }\end{array}$ & $\begin{array}{l}\text { Mean RR shorter during pain and AN than } \\
\text { during baseline. SDNN shorter during } \\
\text { pain than during baseline. }\end{array}$ \\
\hline $\begin{array}{l}\text { Schneider } \\
(2020)[85]\end{array}$ & Germany & $40(20 / 20)$ & $35.1,24-55$ & $\begin{array}{l}\text { Thermal pain: } \\
\text { heat (hot } \\
\text { immersion test) }\end{array}$ & $\begin{array}{l}\text { PTo, PI, and PU on } \\
\text { an NRS }(0-10)\end{array}$ & $\begin{array}{l}\text { The effects of essential oil } \\
\text { inhaler on pain perception }\end{array}$ & $\begin{array}{l}\text { RMSSD } \\
\text { SDNN }\end{array}$ & $\begin{array}{l}\text { RMSSD: significantly higher during pain } \\
\text { than during baseline; higher in the } \\
\text { verum condition. } \\
\text { SDNN: larger in the verum condition than } \\
\text { in the placebo condition. }\end{array}$ \\
\hline $\begin{array}{l}\text { Sclocco et al., } \\
\text { (2016) [86] }\end{array}$ & $\begin{array}{l}\text { United } \\
\text { States }\end{array}$ & $11(3 / 8)$ & $33(4)$ & $\begin{array}{l}\text { Pressure pain } \\
\text { (pressure cuff) }\end{array}$ & $\begin{array}{l}\text { PI on a scale } \\
\quad(0-100)\end{array}$ & $\begin{array}{c}\text { Investigating specific } \\
\text { brainstem nuclei involved } \\
\text { in autonomic responses } \\
\text { to pain }\end{array}$ & $\begin{array}{c}\text { LF } \\
\text { HF } \\
\text { LF/HF ratio }\end{array}$ & $\begin{array}{l}\text { HF power decreased during pain } \\
\text { compared with rest. }\end{array}$ \\
\hline $\begin{array}{l}\text { Sharma et al., } \\
\text { (2017) [87] }\end{array}$ & India & $30(15 / 15)$ & $18-25$ & Cold pain & PTh, PTo & $\begin{array}{l}\text { The modulating role of } \\
\text { slow deep breathing on } \\
\text { pain perception } \\
\text { and cardiac } \\
\text { autonomic activity }\end{array}$ & $\begin{array}{c}\text { Mean RR } \\
\text { SDNN } \\
\text { RMSSD } \\
\text { pNN50 } \\
\text { LF power } \\
\text { HF power } \\
\text { LF/HF ratio }\end{array}$ & $\begin{array}{l}\text { PTo, SDNN, RMSSD, LF power, and } \\
\text { LF/HF ratio significantly higher during } \\
\text { SDB condition compared with } \\
\text { spontaneous breathing. HF power } \\
\text { significantly lower during SDB condition. }\end{array}$ \\
\hline
\end{tabular}


Table 1. Cont.

\begin{tabular}{|c|c|c|c|c|c|c|c|c|}
\hline $\begin{array}{l}\text { Author } \\
\text { (Year) }\end{array}$ & Country & $\mathbf{N}(\mathrm{F} / \mathrm{M})$ & $\begin{array}{c}\text { Age Mean } \\
\text { (SD), } \\
\text { Range }\end{array}$ & $\begin{array}{l}\text { Method of Pain } \\
\text { Induction }\end{array}$ & Pain Assessment & Main Study Focus & $\begin{array}{l}\text { Derived HRV } \\
\text { Measures }\end{array}$ & Hrv and Pain-Related Findings \\
\hline $\begin{array}{l}\text { Streff et al., } \\
(2010)[88]\end{array}$ & Luxemburg & $35(18 / 17)$ & $24,19-57$ & $\begin{array}{l}\text { Heat pain; } \\
\text { cold pressor trial }\end{array}$ & $\begin{array}{l}\text { PTh; PI on a NRS } \\
(0-100) ; \text { PU on a } \\
\text { VAS }(0-10)\end{array}$ & $\begin{array}{l}\text { The physiological effects } \\
\text { of two different tonic } \\
\text { thermal stimuli }\end{array}$ & $\mathrm{LF} / \mathrm{HF}$ ratio & $\begin{array}{l}\text { LF/HF ratio relative to baseline higher on } \\
\text { CPT compared with HIT. }\end{array}$ \\
\hline $\begin{array}{l}\text { Terkelsen et al., } \\
\text { (2004) [89] }\end{array}$ & .'Denmark & $26(0 / 26)$ & $24,21-31$ & $\begin{array}{c}\text { Electrical } \\
\text { stimulation (sural } \\
\text { nerve stimulation) }\end{array}$ & $\begin{array}{l}\text { PTh; PI and PU on } \\
\text { an NRS }(0-10)\end{array}$ & $\begin{array}{l}\text { The effects of mental } \\
\text { stress on pain perception, } \\
\text { HRV, and nociceptive } \\
\text { withdrawal reflex }\end{array}$ & $\begin{array}{l}\text { Mean RR } \\
\text { SDNN } \\
\text { LF } \\
\text { HF }\end{array}$ & $\begin{array}{l}\text { Pain + PASAT decreased mean RR, SDNN, } \\
\text { LF power, CCV-LF, HF power, and } \\
\text { CCV-HF compared with pain at baseline. } \\
\text { Pain + attention decreased HF power. }\end{array}$ \\
\hline $\begin{array}{l}\text { Terkelsen et al., } \\
(2005)[90]\end{array}$ & ·'Denmark & $26(0 / 26)$ & $24,21-31$ & $\begin{array}{c}\text { Electrical } \\
\text { stimulation (sural } \\
\text { nerve stimulation) }\end{array}$ & $\begin{array}{l}\text { PTh; PI on an } \\
\text { NRS }(0-10)\end{array}$ & $\begin{array}{c}\text { The effects of stress on the } \\
\text { HRV responses to } \\
\text { acute pain }\end{array}$ & $\begin{array}{l}\text { Mean RR } \\
\text { SDNN } \\
\text { LF } \\
\text { HF }\end{array}$ & $\begin{array}{c}\text { Pain at rest: mean RR significantly } \\
\text { decreased, LF power and } \\
\text { CCV-LF increased. } \\
\text { Attention to pain: mean RR decreased and } \\
\text { CCV-LF increased. Pain + PASAT: mean } \\
\text { RR decreased. }\end{array}$ \\
\hline $\begin{array}{l}\text { Terkelsen et al., } \\
\text { (2008) [91] }\end{array}$ & · Denmark & $45(22 / 23)$ & $23,18-27$ & $\begin{array}{l}\text { Cold pain; } \\
\text { heat pain; } \\
\text { pressure pain }\end{array}$ & PTh & $\begin{array}{l}\text { The effects of the forearm } \\
\text { immobilization on } \\
\text { pain perception }\end{array}$ & $\begin{array}{l}\text { Mean RR } \\
\text { SDNN } \\
\text { LF } \\
\text { HF }\end{array}$ & $\begin{array}{l}\text { PASAT reduced mean RR, SDNN HF } \\
\text { power, and LF power. }\end{array}$ \\
\hline $\begin{array}{l}\text { Tian et al., } \\
(2020)[92]\end{array}$ & China & $57(30 / 27)$ & $\begin{array}{c}20.28(2.38) \\
19-33\end{array}$ & Cold pain & PI on a scale $(0-10)$ & $\begin{array}{l}\text { The impact of the heart } \\
\text { rate variability on the } \\
\text { relationship between } \\
\text { self-compassion and pain }\end{array}$ & $\mathrm{HF}$ & $\begin{array}{l}\text { Self-compassion was associated with } \\
\text { increased pain when HF was lower; } \\
\text { self-compassion was associated with lower } \\
\text { pain when HF was higher. }\end{array}$ \\
\hline $\begin{array}{l}\text { Tousignant- } \\
\text { Laflamme } \\
\text { and } \\
\text { Marchand } \\
\text { (2009) [93] }\end{array}$ & Canada & $32(32 / 0)$ & $34.3(7.5)$ & Cold pressor test & $\begin{array}{c}\text { PI on a } \\
\text { NRS }(0-100)\end{array}$ & $\begin{array}{l}\text { Autonomic reactivity to } \\
\text { pain throughout the } \\
\text { menstrual cycle }\end{array}$ & $\begin{array}{c}\text { LF } \\
\text { HF } \\
\text { LF/HF ratio } \\
\text { NN50 }\end{array}$ & $\begin{array}{l}\text { No significant differences in HRV were } \\
\text { found between rest and CPT. }\end{array}$ \\
\hline $\begin{array}{l}\text { Tracy et al., } \\
\text { (2018a) [24] }\end{array}$ & Australia & $51(26 / 25)$ & $21.9,18-36$ & Heat pain & PTh & $\begin{array}{l}\text { Sex differences in the } \\
\text { association between } \\
\text { resting HRV and pain } \\
\text { sensitivity }\end{array}$ & $\begin{array}{l}\ln \mathrm{RMSSD} \\
\operatorname{lnLF} \\
\operatorname{lnHF}\end{array}$ & $\begin{array}{l}\text { Higher resting LF was associated with } \\
\text { higher PTh. In men, significant positive } \\
\text { relationship between PTh and resting LF } \\
\text { and HF found. }\end{array}$ \\
\hline $\begin{array}{l}\text { Tracy et al., } \\
(2018 b)[94]\end{array}$ & Germany & $35(29 / 6)$ & $22.80(2.45)$ & Cold pain & $\begin{array}{l}\text { PTh; PTo; PI on a } \\
\text { VAS }(0-10)\end{array}$ & $\begin{array}{l}\text { The association between } \\
\text { HRV and pain sensitivity }\end{array}$ & $\begin{array}{l}\text { LF } \\
\text { HF }\end{array}$ & LF and HF predicted PI. \\
\hline
\end{tabular}


Table 1. Cont.

\begin{tabular}{|c|c|c|c|c|c|c|c|c|}
\hline $\begin{array}{l}\text { Author } \\
\text { (Year) }\end{array}$ & Country & $\mathbf{N}(\mathrm{F} / \mathrm{M})$ & $\begin{array}{c}\text { Age Mean } \\
\text { (SD), } \\
\text { Range }\end{array}$ & $\begin{array}{l}\text { Method of Pain } \\
\text { Induction }\end{array}$ & Pain Assessment & Main Study Focus & $\begin{array}{l}\text { Derived HRV } \\
\text { Measures }\end{array}$ & Hrv and Pain-Related Findings \\
\hline $\begin{array}{l}\text { Treister et al., } \\
\text { (2012) [95] }\end{array}$ & Israel & $55(21 / 34)$ & $\begin{array}{c}25.9(4.1) \\
20-37\end{array}$ & Heat pain & $\begin{array}{c}\text { PTh; PI on an } \\
\text { NPS }(0-100)\end{array}$ & $\begin{array}{l}\text { Comparing different } \\
\text { intensities of pain } \\
\text { employing different } \\
\text { autonomic parameters }\end{array}$ & $\mathrm{HF}$ & $\begin{array}{l}\text { HF showed a negative peak (a decrease } \\
\text { compared with pre-stimulus) followed by } \\
\text { a gradual increase. }\end{array}$ \\
\hline $\begin{array}{l}\text { Van Den } \\
\text { Houte et al., } \\
\text { (2018) [29] }\end{array}$ & Belgium & $63(48 / 15)$ & $\begin{array}{l}21.49(3.80) \\
18-41\end{array}$ & Heat pain & $\begin{array}{l}\text { PTh; PI on an } \\
\text { NRS }(0-100)\end{array}$ & $\begin{array}{l}\text { The association of HRV } \\
\text { and negative affectivity in } \\
\text { the endogenous pain } \\
\text { modulation }\end{array}$ & RMSSD & $\begin{array}{l}\text { Baseline RMSSD significantly related to } \\
\text { the difference in PI between the constant } \\
\text { and offset condition. Higher RMSSD and } \\
\text { larger offset analgesia. }\end{array}$ \\
\hline $\begin{array}{l}\text { Walter et al., } \\
\text { (2014) [96] }\end{array}$ & Germany & $90(45 / 45)$ & $18-65$ & Heat pain & PTh; PTo & $\begin{array}{l}\text { The quantification of pain } \\
\text { experience using } \\
\text { autonomic parameters }\end{array}$ & IBI & $\begin{array}{c}\text { An association between pain and IBI } \\
\text { was found. }\end{array}$ \\
\hline $\begin{array}{l}\text { Ye et al., } \\
\text { (2017) [97] }\end{array}$ & Taiwan & $40(19 / 21)$ & $22.5,20-27$ & Heat pain & PI on an NRS & $\begin{array}{l}\text { Changes in physiological } \\
\text { parameters during the } \\
\text { process of pain } \\
\text { production and relief }\end{array}$ & $\begin{array}{l}\text { Mean RR } \\
\text { LF } \\
\text { HF }\end{array}$ & $\begin{array}{l}\text { LF significantly changed between } \\
\text { segments D and E. }\end{array}$ \\
\hline
\end{tabular}




\section{Results}

Demographic Characteristics of the Sample

The selected studies were conducted from 2004 [59] to 2020 [32], including a total sample of 6364 participants with percentages of $55.7 \%$ females and $44.3 \%$ males. In three studies, the percentage or the number of women and men was not reported [38,51,61]. Some studies adopted a sample that included exclusively female $[39,40,49,50,74,82,84,93]$ or male participants $[57-59,62,64,77,78,89,90]$. The average age of the participants ranged from 18.98 [52] to 73.44 years [41,42]. The largest range was 18-84 [41].

The selected studies were conducted in Europe $(\mathrm{N}=38)$, the United States $(\mathrm{N}=12)$, Israel $(\mathrm{N}=5)$, Canada $(\mathrm{N}=5)$, Australia $(\mathrm{N}=4)$, India $(\mathrm{N}=1)$, Taiwan $(\mathrm{N}=1)$, Brazil $(\mathrm{N}=1)$, China $(\mathrm{N}=2)$, Japan $(\mathrm{N}=1)$, and Korea $(\mathrm{N}=1)$. Except for a study conducted by Kim et al. in 2019 [65], all studies were conducted with homogeneous samples for ethnicity and nationality. All studies adopted a cross-sectional design.

HRV Measurement

In all studies, HRV measurement was conducted by a continuous ECG recording, which lasted at least $5 \mathrm{~min}$ as recommended by the guidelines of the European Society of Cardiology and the North American society [99]. Heart rate variability was evaluated considering time domain analyses, frequency domain analyses, or both (see Table 1).

Effects of Different Methods of Pain Induction on HRV

Thermal Stimuli $(\mathrm{N}=51)$

Thermal stimuli (i.e., cold and heat) were the most adopted method of pain induction (e.g., [49]).

Cold Pain

Cold pain was elicited using mostly the cold pressor task $[32,39,43,51,52,56,61,67,78,93]$, but it was also induced using a cold plate [13,88], a thermal aluminum cylinder device [83], the immersion of a hand $[41,57,87,94]$ or a foot [72,73] in cold water, facial cooling [62], thermal stimulation devices [60,72,91,98], the cold cup test [50], and holding a plastic bottle with iced water $[68,92]$.

Cold pain stimulation elicited an increase in the parasympathetic components of HRV, such as RMSSD [32,67,78,83,87], SDNN [67,83], CVI [51,87], HF [83], mean RR [83], and IBI [62]. Nevertheless, an increase in the sympathetic activity was registered by the reduction in HRV [41], mean IBI [78], and mean RR [61] and the increase in the log LF $[60,83,87]$ and LF/HF ratio [87,88]. A study by De Pascalis and Scacchia [50] found a negative correlation between pain and the time domain but not between pain and the frequency domain. Bendixen et al. [39] found only a reduction in vagal measures (RMSSD, HF power; CCV-HF). However, a relationship between cold pain and the HRV parameters was not found in two studies [56,93].

Heat Pain

Thermal stimulation devices [24,29,33,36,37,45,48,54,57,58,63,65,70-74,77,80,83,91,95,96,98], a laser [47], an IC thermostat [97], and the immersion of a hand in hot water [88] were adopted to induce heat stimulation.

Although no correlations between pain and HRV were reported $[53,65,80]$, other authors underlined an association $[70,96]$. Both sympathetic and parasympathetic activity changes due to the heat stimuli were reported. On the one hand, an increase in sympathetic activity [48] expressed by the LF/HF ratio [36,47,54] and LF [47] and a decrease in RR [47], lnSDNN [71], and HF [95] was evidenced. On the other hand, a parasympathetic increase was found [74], indexed by the increase in HF [33] and RMSSD [85] and the decrease in lnLF [71]. Aslaksen and Flaten [37] showed that placebo administration before painful exposure reduced the LF/HF ratio after the painful heat stimulation, suggesting that placebo administration can affect the pain experience, reducing physiological stress [37].

Mechanical Stimuli $(\mathrm{N}=18)$

Pressure pain was elicited by digital pressure algometers $[33,38,42,49,57,65,78,79,81,82,84,91]$, pressing the nail bed with a spring-loaded device [76], and inflating a pressure cuff on the lower leg [86]. Some studies [81,84,86] found higher sympathetic activity during pain, indexed by the decrease in SDNN [84] and HF and the increase in LF and the LF/HF ratio. 
On the contrary, other authors showed predominant parasympathetic activity in responses to a painful pressure stimulus $[38,49,73]$. Finally, two studies reported no relationship between pressure pain and the HRV parameters [42,65].

Four studies adopted pinprick stimuli in order to induce experimental mechanical pain, using a set of probes [65] or a von Frey filament [72-74,83]. A relationship between parasympathetic activity and pain perception was evidenced.

Electrical Stimuli $(\mathrm{N}=9)$

Electrical stimulation was induced via electrical stimulators $[35,46,49,56,64,80]$. In two studies $[89,90]$, sural nerve stimulation was delivered via solid gel surface electrodes. Courtois et al. [49] found an increase in RMSSD during pain while the participants practiced slow deep breathing. Ghione et al. [59] induced pain by exposing participants to an electromagnetic field. The LF component increased during both sham and magnetic exposure, while the HF component remained constant during real exposure but increased during the sham condition. A reduction in HRV was found in two studies [46,64]. Similarly, sympathetic activation increased when the pain was elicited both during mental arithmetic stress and at rest [89,90]. Piovesan et al. [80] registered an increase in the HF components. Finally, the authors of [56] found no relationship between pain and HRV.

Injection of Hypertonic Saline Solutions ( $\mathrm{N}=5)$

A sterile hypertonic saline solution (5\%) was injected to induce experimental masseter muscle pain $[39,40]$ and deep and superficial pain [44], and a hypertonic saline solution $(7 \%)[55,66]$ was injected to induce experimental muscle pain. Two studies found an increase in sympathetic activity during the infusion [55] in both deep and superficial pain [44]. However, parasympathetic parameters such as RMSSD and HF were higher when the only pain stimulation was the solution injection, rather than a condition in which muscle pain was associated with a CPT [39] or with a PASAT [40]. Only one study found no correlation between pain and HRV [66].

Visceral Pain: Esophageal Balloon Distension $(\mathrm{N}=2)$

Two studies focused on visceral pain $[8,76]$ while adopting esophageal painful balloon distension. Interestingly, one study [8] found that the participants that were classified as "neurotic-introvert" had an increased parasympathetic activity expressed by CVCna in response to pain, while participants classified as "extrovert-emotionally stable" had a high resting CVCna and withdrawal from it during pain stimulation. Others [76] found that both the sympathetic and parasympathetic branches were activated by visceral and somatic pain.

Bed of Nails $(\mathrm{N}=1)$

One study elicited a painful sensation by letting participants lie on a bed of nails. Stimulation consisted of a soft cotton fabric case filled with a foam rubber rectangle [75]. The authors found an increase in parasympathetic activity expressed by HF when participants were lying on the bed of nails.

Relationship between Subjective Pain Measures and HRV

Subjective Pain Measurement

Subjective measures of pain perception, such as pain intensity, pain unpleasantness, pain thresholds, and pain tolerance, were assessed (see Table 1).

Pain thresholds were assessed by adopting different methods. Regarding heat stimuli, pain thresholds were assessed by increasing the temperature of the device until the subject perceived the stimulus as painful $[24,29,45,58,63,65,72-74,85,88,91,94-96,98]$ or considered the temperature estimated as painful at a specific point on a VAS. Then, the results were averaged for the number of trials $[13,47,53,77]$ with the calculated thresholds being the average temperature (in ${ }^{\circ} \mathrm{C}$ ) at which each participant indicated experiencing noticeable pain and moderate pain during each of the three exposures. Cold pain thresholds were identified as the cold temperature at which the subject reported the stimulus as painful $[87,88,91,98]$ or the total time from immersion until a participant verbally reported pain [67]. Pressure pain thresholds were reached when the participant's sensation changed from pressure (evoked via a pressure algometer) to pain, which was averaged for a specific number of 
trials $[34,42,65,76,78,79,81,82,91]$. The pain thresholds for the electrical stimulation were obtained when the subject reported a pain sensation evoked by the current $[35,46,89,90]$. The first pain sensation evoked by the balloon distension was defined as the visceral pain threshold $[8,76]$.

Pain tolerance was assessed by considering when the level of heat $[45,58,85,96]$, cold $[87,94]$, pressure $[76,78,81,82]$, or visceral pain $[8,76]$ became unbearable for the participant. Another method to assess cold pain tolerance was calculating the total time in seconds that the participant's hand was completely submerged in the water $[52,67]$.

Relationship with HRV

The relationship between the subjective pain measures and HRV was reported [24,52,81]. Two studies highlighted a positive relationship between heat pain thresholds and resting LF $[13,24]$. A higher LF-HRV was positively correlated to a higher temperature at which the subject started to perceive the heat stimulus as painful. Conversely, pain unpleasantness was lower in higher resting LF-HRV [13]. Pain tolerance was associated with higher parasympathetic activity expressed by the HRV (i.e., increase in HF-HRV and decrease in the LF/HF HRV ratio) [52,82].

Recent studies found a negative correlation between parasympathetic activity and the pain intensity ratings [57,68,92]. Accordingly, slow deep breathing and HR biofeedback [45] could reduce both the pain intensity and sympathetic activity expressed by a higher SDNN [45]. Furthermore, placebo analgesia produced an increase in the time domains of HRV and greater pain relief [50]. Pain intensity was also negatively correlated with HRV when the participants had greater parasympathetic activity during the recovery phase [51]. Parasympathetic activity correlated with a more efficient pain modulation capacity [72-74].

No correlation between subjective pain measures and HRV was reported or found by many studies (see Table 1).

\section{Discussion}

The main aim of this study was to investigate the relationship between pain and heart rate variability, summarizing the results of experimental studies that induced pain in healthy adult samples. In recent years, an increasing number of studies have adopted HRV as a physiological index of the organism's ability to provide a flexible response to stress, such as pain. The Vagal Tank Theory [100], relying on the Neurovisceral Integration Model [17], highlights the role of the vagus nerve in the control of cardiac activity and in goal-directed behavior, as well as in the individual self-regulation ability [100]. The Neurovisceral Integration Model $[17,19]$ assumes that the goal-directed behavior and the self-regulation ability of an organism are structurally and functionally supported by the Central Autonomic Network [18], a complex network of brain structures whose primary output is the cardiac vagal control expressed by HRV. Accordingly, our study confirms a relationship between the autonomic nervous system indexed by HRV and the pain response to nociceptive stimulation. Our findings can be reported in two themes: (1) how the autonomic nervous system reacts to pain and (2) how the autonomic nervous system is associated with subjective pain perception.

For the first issue, generally, the studies included in the systematic review reported a significant change in HRV during pain induction. The main finding about the autonomic response to pain is an increase in sympathetic activity (e.g., [36,54]), according to the previous review conducted by Koenig et al. [14]. Evidence suggests that this response is independent of the adopted method of pain induction. Burton et al. [44] found that both deep and cutaneous pain elicit an increase in the LF/HF ratio, in addition to Chouchou et al. [47] underlining a sympathetic activation to heat pain during sleep. The same sympathetic increase was found in response to cold pain, (e.g., [39,60]).

Nevertheless, different circumstances can increase the vagal activity expressed by the parasympathetic components of HRV [45]. For example, different techniques of breathing, such as slow deep breathing [49] or meditation, could reduce vagal withdrawal during pain. Adler-Neal et al. [33] focused on the autonomic responses to pain while mindfulness 
meditation or sham meditation were practiced. They found a parasympathetic increase during both meditation techniques, possibly due to the slow breathing. Similar results were obtained by Chalaye et al. [45], where slow deep breathing patterns increased vagal activity compared with normal breathing. Cotton et al. [48] found that yoga practitioners for at least 6 years had the same parasympathetic activity during a warm stimulation and a painful one compared with the control group, which reported a withdrawal of vagal activity during the painful stimulation, despite similar pain ratings.

Another aspect that could influence an autonomic response to pain is a drug or medication assumption. Some studies reported that the administration of substances with analgesic effects, such as propranolol [78] or clonidine [73], can increase parasympathetic activity during pain. However, a placebo [50] also generates a similar response. The findings suggest that placebo analgesia, induced administering a placebo, can increase HRV and induce pain relief $[37,50]$, consistent with the Neurovisceral Integration Model $[19,25]$. Furthermore, autonomic responses to the same painful stimulation can differ depending on the affective states [32] or the empathetic or unempathetic context [53,54].

Another result evidenced by the review is a parasympathetic activation during painful stimulations. Activation of both the sympathetic and parasympathetic branches of the autonomic nervous system appears to be counterintuitive, but the Vagal Tank Theory [100] can explain it. The autonomic nervous system's reaction to painful stimulation is complex. A large or small vagal withdrawal depends on the level of activity required by the situation and how much top-down executive processing is needed to face the situation [100]. Moreover, the autonomic reaction to pain could be affected by several factors, such as ethnicity, sex, age, body mass index, breathing patterns, the intensity of the stimulation, or the affective state that could influence results [24].

Regarding the second issue, an association between the autonomic nervous system and the subjective experience of pain was found [68]. Higher parasympathetic activity is associated with better self-regulation capacities and, accordingly, a higher pain inhibition capacity $[50,52,68]$. In this field, an interesting result was related to self-compassion abilities [68,92] that appear to be associated with high HF and lower pain ratings. Tian et al. [92] explained that self-compassion means to treat oneself with kindness and acceptance, and it seems that this ability enhances a better bodily control over pain-related arousal and a better subjective pain experience [92].

Most studies have underlined that when the parasympathetic component of $\mathrm{HRV}$ is high, pain relief or a better management of painful situations (e.g., pain tolerance) occur. The inhibitory vagal effect on pain could be responsible for these results. This hypothesis is in line with the Neurovisceral Integration Model, and it is supported by the strong positive relationship between vagal activation and the prefrontal cortex, as highlighted by Perlaki et al. [77]. Finally, Appelhans and Luecken [13] found a negative association between the sympathetic activity expressed by the LF components and pain sensitivity but not between HF and pain. According to the authors, this finding is explicable because LF has a complex association with the arterial baroreflex, a homeostatic mechanism mediated by the autonomic nervous system, whose components also mediate an endogenous pain inhibitory pathway [13]. These results are consistent with a recent study [60] that identified a three-way relationship between HRV, cortical regions underpinning pain processing, and subjective pain experience. In particular, the connectivity between the periaqueductal gray (PAG) $[18,25]$ and the ventromedial prefrontal cortex (vmPFC) at rest was associated with high LF during painful stimulation and lower pain ratings. The PAG receives nociceptive inputs and is involved in descending nociceptive modulation [18,60].

Despite the important update to the study of Koenig et al. [14] and the new evidence reported, this review has some limitations. The strict selection criteria excluded works that may contain relevant information about the activity of ANS in response to pain. Many of the studies included were conducted by the same group of authors, and this could have introduced biases influenced by the theoretical background of those who led the study. However, the selected studies may well represent the state of the knowledge. Moreover, the 
lack of quantitative analysis (i.e., meta-analysis) lowers the strength of our inferences by not furnishing an effect size for the studies. Another limitation could be indirectly linked to the publication bias. The choice to include only academic articles published in peer-reviewed journals may have limited the selection to only those studies that have obtained results in line with the literature. As a consequence, the results may overestimate this relationship. Moreover, the choice to select only studies published in English could have led to the elimination of studies conducted on other populations and written in different languages, further limiting the generalizability of the results. Finally, the lack of an unambiguous subjective measure of pain sensitivity makes the results heterogeneous. Further studies could develop an instrument to measure pain sensitivity and better define constructs related to pain perception. For example, it could be better to assess pain intensity separately from pain unpleasantness in order to assess the sensory and emotional components more precisely and relate how each component interacts with autonomic activity. Moreover, the role of cognitive functions such as inhibition could be evaluated, considering its association with both HRV and pain.

\section{Conclusions}

According to our results, we can conclude that HRV is a good measure of autonomic reactivity to nociceptive stimulation. Studies that have investigated changes in HRV in response to pain reported changes in autonomic response, both in sympathetic and parasympathetic branches. Our summarized evidence may be helpful for further research and have important clinical implications. Since HRV appears to be impaired in several chronic pain conditions that can worsen the quality of life, future researchers can take advantage of the use of HRV. According to our results, many practices (e.g., yoga and mindfulness) or drugs can increase vagal activity. For this reason, HRV can be a reliable index to assess the efficacy of treatments on pain management in clinical populations. Moreover, it could be tested if techniques of control over HRV such as HRV biofeedback, which were demonstrated to be effective in improving cognitive functions and stress management [101], can increase pain relief or pain management in clinical samples. Further studies are needed to overcome the limitations and also better understand this relationship in the large variety of health conditions associated both with ANS changes and pain (i.e., chronic pain).

Author Contributions: Conceptualization and writing—original draft preparation: G.F., G.T., M.C.; critical revision of the article: G.F., G.T., M.P., V.D.P. and M.C; funding acquisition: M.P., M.C. All authors have read and agreed to the published version of the manuscript.

Funding: This research was funded by the Italian Ministry of Health, grant number RF-2018-12365682.

Conflicts of Interest: The authors declare no conflict of interest.

\section{References}

1. Raja, S.N.; Carr, D.B.; Cohen, M.; Finnerup, N.B.; Flor, H.; Gibson, S.; Keefe, F.J.; Mogil, J.S.; Ringkamp, M.; Vader, K. The revised international association for the study of pain definition of pain: Concepts, challenges, and compromises. Pain 2020, 161, 1976-1982. [CrossRef] [PubMed]

2. IASP, I. Subcommittee on taxonomy. Pain terms. A list with definitions and notes on usage. Pain 1979, 6, $249-252$.

3. Lumley, M.A.; Cohen, J.L.; Borszcz, G.S.; Cano, A.; Radcliffe, A.M.; Porter, L.S.; Schubiner, H.; Keefe, F.J. Pain and emotion: A biopsychosocial review of recent research. J. Clin. Psychol. 2011, 67, 942-968. [CrossRef] [PubMed]

4. Kumar, K.H.; Elavarasi, P. Definition of pain and classification of pain disorders. J. Adv. Clin. Res. Insights 2016, 3, 87-90. [CrossRef]

5. Ellison, D.L. Physiology of pain. Crit. Care Nurs. Clin. 2017, 29, 397-406. [CrossRef] [PubMed]

6. Hudspith, M.J.; Siddall, P.J.; Munglani, R. Physiology of pain. In Fundamentals of Anaesthesia; Cambridge University Press: Cambridge, UK, 2006; pp. 267-285.

7. Chu, Y.; Zhao, X.; Han, J.; Su, Y. Physiological signal-based method for measurement of pain intensity. Front. Neurosci. 2017, 11, 279. [CrossRef] [PubMed]

8. Paine, P.; Worthen, S.F.; Gregory, L.J.; Thompson, D.G.; Aziz, Q. Personality differences affect brainstem auto-nomic responses to visceral pain. Neurogastroenterol. Motil. 2009, 21, 1155-e98. [CrossRef] 
9. Cruccu, G.; Anand, P.; Attal, N.; Garcia-Larrea, L.; Haanpää, M.; Jørum, E.; Serra, J.; Jensen, T.S. EFNS guidelines on neuropathic pain assessment. Eur. J. Neurol. 2004, 11, 153-162. [CrossRef]

10. Huskisson, E.C. Measurement of pain. Lancet 1974, 304, 1127-1131. [CrossRef]

11. Gracely, R.H.; McGrath, P.; Dubner, R. Ratio scales of sensory and affective verbal pain descriptors. Pain 1978, 5, 5-18. [CrossRef]

12. Bourne, S.; Machado, A.G.; Nagel, S.J. Basic anatomy and physiology of pain pathways. Neurosurg. Clin. N. Am. 2014, 25, 629-638. [CrossRef] [PubMed]

13. Craig, A. A new view of pain as a homeostatic emotion. Trends Neurosci. 2003, 26, 303-307. [CrossRef]

14. Koenig, J.; Jarczok, M.N.; Ellis, R.; Hillecke, T.; Thayer, J. Heart rate variability and experimentally induced pain in healthy adults: A systematic review. Eur. J. Pain 2013, 18, 301-314. [CrossRef] [PubMed]

15. Thayer, J.F.; Lane, R.D. A model of neurovisceral integration in emotion regulation and dysregulation. J. Affect. Disord. 2000, 61, 201-216. [CrossRef]

16. Thayer, J.F.; Lane, R.D. The role of vagal function in the risk for cardiovascular disease and mortality. Biol. Psychol. 2007, 74, 224-242. [CrossRef]

17. Benarroch, E.E. Pain-autonomic interactions. Neurol. Sci. 2006, 27, s130-s133. [CrossRef]

18. Thayer, J.F.; Åhs, F.; Fredrikson, M.; Sollers, J.J.; Wager, T.D. A meta-analysis of heart rate variability and neu-roimaging studies: Implications for heart rate variability as a marker of stress and health. Neurosci. Biobehav. Rev. 2012, 36, 747-756. [CrossRef]

19. Forte, G.; Morelli, M.; Grässler, B.; Casagrande, M. Decision making and Heart Rate variability: A systematic review. Appl. Cogn. Psychol. 2021, 36, 100-110. [CrossRef]

20. Forte, G.; Favieri, F.; Casagrande, M. Heart rate variability and cognitive function: A systematic review. Front. Neurosci. 2019, 13, 710. [CrossRef]

21. Forte, G.; Morelli, M.; Casagrande, M. Heart rate variability and decision-making: Autonomic responses in making decisions. Brain Sci. 2021, 11, 243. [CrossRef]

22. Forte, G.; Favieri, F.; Oliha, E.O.; Marotta, A.; Casagrande, M. Anxiety and attentional processes: The role of resting heart rate variability. Brain Sci. 2021, 11, 480. [CrossRef] [PubMed]

23. Laborde, S.; Mosley, E.; Thayer, J.F. Heart rate variability and cardiac vagal tone in psychophysiological research-recommendations for experiment planning, data analysis, and data reporting. Front. Psychol. 2017, 8, 213. [CrossRef] [PubMed]

24. Tracy, L.M.; Koenig, J.; Georgiou-Karistianis, N.; Gibson, S.J.; Giummarra, M.J. Heart rate variability is associated with thermal heat pain threshold in males, but not females. Int. J. Psychophysiol. 2018, 131, 37-43. [CrossRef] [PubMed]

25. Benarroch, E.E. Pain-autonomic interactions: A selective review. Clin. Auton. Res. 2001, 11, 343-349. [CrossRef] [PubMed]

26. Bruehl, S.; Chung, O.Y. Interactions between the cardiovascular and pain regulatory systems: An updated review of mechanisms and possible alterations in chronic pain. Neurosci. Biobehav. Rev. 2004, 28, 395-414. [CrossRef]

27. Nielsen, R.; Nikolajsen, L.; Krøner, K.; Mølgaard, H.; Vase, L.; Jensen, T.S.; Terkelsen, A.J. Pre-operative baroreflex sensitivity and efferent cardiac parasympathetic activity are correlated with post-operative pain. Acta Anaesthesiol. Scand. 2014, 59, 475-485. [CrossRef]

28. Bruehl, S.; Chung, O.Y.; Jirjis, J.N.; Biridepalli, S. Prevalence of clinical hypertension in patients with chronic pain compared to nonpain general medical patients. Clin. J. Pain 2005, 21, 147-153. [CrossRef] [PubMed]

29. Van Den Houte, M.; Van Oudenhove, L.; Bogaerts, K.; Van Diest, I.; Van den Bergh, O. Endogenous pain modulation: Association with resting heart rate variability and negative affectivity. Pain Med. 2018, 19, 1587-1596. [CrossRef] [PubMed]

30. Liberati, A.; Altman, D.G.; Tetzlaff, J.; Mulrow, C.; Gøtzsche, P.C.; Ioannidis, J.P.A.; Clarke, M.; Devereaux, P.J.; Kleijnen, J.; Moher, D. The PRISMA statement for reporting systematic reviews and meta-analyses of studies that evaluate health care interventions: Explanation and elaboration. PLoS Med. 2009, 62, e1-e34. [CrossRef]

31. Moher, D.; Liberati, A.; Tetzlaff, J. Linee guida per il reporting di revisioni sistematiche e meta-analisi: Il PRISMA Statement. PLoS Med 2009, 6, e1000097. [CrossRef]

32. Acevedo, A.M.; Leger, K.A.; Jenkins, B.N.; Pressman, S.D. Keep calm or get excited? Examining the effects of differ-ent types of positive affect on responses to acute pain. J. Posit. Psychol. 2020, 1-10. [CrossRef]

33. Adler-Neal, A.L.; Waugh, C.E.; Garland, E.L.; Shaltout, H.A.; Diz, D.I.; Zeidan, F. The role of heart rate variability in mindfulnessbased pain relief. J. Pain 2020, 21, 306-323. [CrossRef] [PubMed]

34. de Araujo, F.X.; Schell, M.S.; Ferreira, G.E.; Pessoa, M.D.V.; de Oliveira, L.R.; Borges, B.G.; Mecagnan, F.E.; Plentz, R.D.M.; Silva M.F. Auto-nomic function and pressure pain threshold following thoracic mobilization in asymptomatic subjects: A randomized controlled trial. J. Bodyw. Mov. Ther. 2018, 22, 313-320. [CrossRef] [PubMed]

35. Arsenault, M.; Ladouceur, A.; Lehmann, A.; Rainville, P.; Piché, M. Pain modulation induced by respiration: Phase and frequency effects. Neuroscience 2013, 252, 501-511. [CrossRef]

36. Aslaksen, P.M.; Myrbakk, I.N.; Høifødt, R.S.; Flaten, M.A. The effect of experimenter gender on autonomic and subjective responses to pain stimuli. Pain 2007, 129, 260-268. [CrossRef]

37. Aslaksen, P.M.; Flaten, M.A. The roles of physiological and subjective stress in the effectiveness of a placebo on experimentally induced pain. Psychosom. Med. 2008, 70, 811-818. [CrossRef]

38. Balocchi, R.; Varanini, M.; Menicucci, D.; Santarcangelo, E.L.; Migliorini, S.; Fontani, G.; Carli, G. Heart rate variability in subjects with different hypnotic susceptibility receiving nociceptive stimulation and suggestions of analge-sia. In Proceedings of the 2005 IEEE Engineering in Medicine and Biology 27th Annual Conference, Shanghai, China, 17-18 January 2006; pp. 6996-6999. 
39. Bendixen, K.H.; Terkelsen, A.J.; Baad-Hansen, L.; Cairns, B.E.; Svensson, P. Experimental stressors alter hyper-tonic saline-evoked masseter muscle pain and autonomic response. J. Orofac. Pain 2012, 26, 191-205.

40. Bendixen, K.H.; Terkelsen, A.J.; Baad-Hansen, L.; E Cairns, B.; Svensson, P. Effect of propranolol on hypertonic saline-evoked masseter muscle pain and autonomic response in healthy women during rest and mental arithmetic task. J. Orofac. Pain 2013, 27, 243-255. [CrossRef]

41. Boggero, I.A.; Segerstrom, S. Maintenance of affective wellbeing following acute pain in healthy older and younger adults. $J$. Behav. Med. 2019, 42, 934-946. [CrossRef]

42. Boggero, I.A.; Segerstrom, S.C. Self-regulatory ability, fatigue, and the experience of pain: Mechanistic insights from pain-free undergraduates. Psychophysiology 2019, 56, e13388. [CrossRef]

43. Bourassa, K.J.; Ruiz, J.M.; Sbarra, D.A. The impact of physical proximity and attachment working models on car-diovascular reactivity: Comparing mental activation and romantic partner presence. Psychophysiology 2019, 56, e13324. [CrossRef]

44. Burton, A.R.; Birznieks, I.; Bolton, P.S.; Henderson, L.A.; Macefield, V.G. Effects of deep and superficial experimentally induced acute pain on muscle sympathetic nerve activity in human subjects. J. Physiol. 2009, 587, 183-193. [CrossRef] [PubMed]

45. Chalaye, P.; Goffaux, P.; Lafrenaye, S.; Marchand, S. Respiratory effects on experimental heat pain and cardiac activity. Pain Med. 2009, 10, 1334-1340. [CrossRef] [PubMed]

46. Cho, S.-H. Frequency and intensity of electrical stimulation of human sympathetic ganglia affect heart rate variability and pain threshold. Appl. Sci. 2019, 9, 4490. [CrossRef]

47. Chouchou, F.; Pichot, V.; Perchet, C.; Legrain, V.; Garcia-Larrea, L.; Roche, F.; Bastuji, H. Autonomic pain responses during sleep: A study of heart rate variability. Eur. J. Pain 2011, 15, 554-560. [CrossRef] [PubMed]

48. Cotton, V.A.; Low, L.A.; Villemure, C.; Bushnell, M.C. Unique Autonomic Responses to Pain in Yoga Practitioners. Psychosom. Med. 2018, 80, 791-798. [CrossRef] [PubMed]

49. Courtois, I.; Gholamrezaei, A.; Jafari, H.; Lautenbacher, S.; Van Diest, I.; Van Oudenhove, L.; Vlaeyen, J.W. Respiratory hypoalgesia? The effect of slow deep breathing on electrocutaneous, thermal, and mechanical pain. J. Pain 2020, 21, 616-632. [CrossRef]

50. De Pascalis, V.; Scacchia, P. The influence of reward sensitivity, heart rate dynamics and EEG-delta activity on placebo analgesia. Behav. Brain Res. 2018, 359, 320-332. [CrossRef]

51. Dodo, N.; Hashimoto, R. The effect of anxiety sensitivity on psychological and biological variables during the cold pressor test. Auton. Neurosci. 2017, 205, 72-76. [CrossRef]

52. Evans, D.R.; Eisenlohr-Moul, T.; Button, D.F.; Baer, R.A.; Segerstrom, S.C. Self-regulatory deficits associated with unpracticed mindfulness strategies for coping with acute pain. J. Appl. Soc. Psychol. 2013, 44, 23-30. [CrossRef]

53. Fauchon, C.; Faillenot, I.; Perrin, A.M.; Borg, C.; Pichot, V.; Chouchou, F.; Garcia-Larrea, L.; Peyron, R. Does an observer's empathy influence my pain? Effect of perceived empathetic or unempathetic support on a pain test. Eur. J. Neurosci. 2017, 46, 2629-2637. [CrossRef] [PubMed]

54. Fauchon, C.; Pichot, V.; Faillenot, I.; Pommier, B.; Garcia-Larrea, L.; Peyron, R.; Chouchou, F. Contextual modulation of autonomic pain reactivity. Auton. Neurosci. 2018, 212, 28-31. [CrossRef] [PubMed]

55. Fazalbhoy, A.; Birznieks, I.; Macefield, V.G. Individual differences in the cardiovascular responses to tonic muscle pain: Parallel increases or decreases in muscle sympathetic nerve activity, blood pressure and heart rate. Exp. Physiol. 2012, 97, 1084-1092. [CrossRef] [PubMed]

56. Fidanza, F.; Varanini, M.; Ciaramella, A.; Carli, G.; Santarcangelo, E.L. Pain modulation as a function of hypnotiz-ability: Diffuse noxious inhibitory control induced by cold pressor test vs explicit suggestions of analgesia. Physiol. Behav. 2017, 171, 135-141. [CrossRef]

57. Geisler, M.; Herbsleb, M.; Bär, K.-J.; Weiss, T. Dissociation of endogenous pain inhibition due to conditioned pain modulation and placebo in male athletes versus nonathletes. Front. Psychol. 2020, 11, 553530. [CrossRef]

58. Geva, N.; Pruessner, J.; Defrin, R. Triathletes lose their advantageous pain modulation under acute psychosocial stress. Med. Sci. Sports Exerc. 2017, 49, 333-341. [CrossRef]

59. Ghione, S.; Del Seppia, C.; Mezzasalma, L.; Emdin, M.; Luschi, P. Human head exposure to a 37 Hz electromagnetic field: Effects on blood pressure, somatosensory perception, and related parameters. Bioelectromagnetics 2004, 25, 167-175. [CrossRef]

60. Hohenschurz-Schmidt, D.J.; Calcagnini, G.; DiPasquale, O.; Jackson, J.B.; Medina, S.; O’Daly, O.; O’Muircheartaigh, J.; Rubio, A.D.L.; Williams, S.C.R.; McMahon, S.B.; et al. Linking pain sensation to the autonomic nervous system: The role of the anterior cingulate and periaqueductal gray resting-state networks. Front. Neurosci. 2020, 14, 147. [CrossRef]

61. Huggins, J.; Rakobowchuk, M. Utility of lacrimal caruncle infrared thermography when monitoring alterations in autonomic activity in healthy humans. Graefe's Arch. Clin. Exp. Ophthalmol. 2018, 119, 531-538. [CrossRef]

62. Iorfino, F.; Alvares, G.; Guastella, A.J.; Quintana, D.S. Cold face test-induced increases in heart rate variability are abolished by engagement in a social cognition task. Psychophysiology 2016, 30, 38-46. [CrossRef]

63. Jafari, H.; Gholamrezaei, A.; Franssen, M.; Van Oudenhove, L.; Aziz, Q.; Bergh, O.V.D.; Vlaeyen, J.W.; Van Diest, I. Can slow deep breathing reduce pain? An experimental study exploring mechanisms. J. Pain 2020, 21, 1018-1030. [CrossRef] [PubMed]

64. Jess, G.; Pogatzki-Zahn, E.M.; Zahn, P.K.; Meyer-Frieem, C.H. Monitoring heart rate variability to assess experimentally induced pain using the analgesia nociception index: A randomised volunteer study. Eur. J. Anaesthesiol. 2016, 33, 118-125. [CrossRef] [PubMed] 
65. Kim, H.J.; Greenspan, J.D.; Ohrbach, R.; Fillingim, R.; Maixner, W.; Renn, C.L.; Johantgen, M.; Zhu, S.; Dorsey, S.G. Racial/ethnic differences in experimental pain sensitivity and associated factors - Cardiovascular responsiveness and psychological status. PLoS ONE 2019, 14, e0215534. [CrossRef]

66. Kobuch, S.; Fazalbhoy, A.; Brown, R.; Macefield, V.G. Inter-individual responses to experimental muscle pain: Baseline physiological parameters do not determine whether muscle sympathetic nerve activity increases or decreases during pain. Front. Neurosci. 2015, 9, 471. [CrossRef]

67. Konstantinou, P.; Trigeorgi, A.; Georgiou, C.; Gloster, A.T.; Panayiotou, G.; Karekla, M. Comparing apples and oranges or different types of citrus fruits? Using wearable versus stationary devices to analyze psychophysiological data. Psychophysiology 2020, 57, e13551. [CrossRef] [PubMed]

68. Luo, X.; Liu, J.; Che, X. Investigating the influence and a potential mechanism of self-compassion on experimental pain: Evidence from a compassionate self-talk protocol and heart rate variability. J. Pain 2019, 21, 790-797. [CrossRef]

69. Martin, S.L.; Kerr, K.L.; Bartley, E.J.; Kuhn, B.L.; Palit, S.; Terry, E.L.; DelVentura, J.L.; Rhudy, J.L. Respiration-induced hypo-algesia: Exploration of potential mechanisms. J. Pain 2012, 13, 755-763. [CrossRef]

70. Matthewson, G.M.; Woo, C.-W.; Reddan, M.C.; Wager, T.D. Cognitive self-regulation influences pain-related physiology. Pain 2019, 160, 2338-2349. [CrossRef]

71. Meeuse, J.J.; Löwik, M.S.; Löwik, S.A.; Aarden, E.; van Roon, A.M.; Gans, R.O.; van Wijhe, M.; Lefrandt, J.D.; Reyners, A.K. Heart rate variability parameters do not correlate with pain intensity in healthy volunteers. Pain Med. 2013, 14, 1192-1201. [CrossRef]

72. Nahman-Averbuch, H.; Dayan, L.; Sprecher, E.; Hochberg, U.; Brill, S.; Yarnitsky, D.; Jacob, G. Sex differences in the relationships between parasympathetic activity and pain modulation. Physiol. Behav. 2016, 154, 40-48. [CrossRef]

73. Nahman-Averbuch, H.; Dayan, L.; Sprecher, E.; Hochberg, U.; Brill, S.; Yarnitsky, D.; Jacob, G. Pain modulation and autonomic function: The effect of clonidine. Pain Med. 2016, 17, 1292-1301. [CrossRef] [PubMed]

74. Nahman-Averbuch, H.; Sprecher, E.; Jacob, G.; Yarnitsky, D. The relationships between parasympathetic function and pain perception: The role of anxiety. Pain Pract. 2016, 16, 1064-1072. [CrossRef] [PubMed]

75. Olsson, E.M.; von Schéele, B. Relaxing on a bed of nails: An exploratory study of the effects on the autonomic, cardiovascular, and respiratory systems, and saliva cortisol. J. Altern. Complement. Med. 2011, 17, 5-12. [CrossRef] [PubMed]

76. Paine, P.; Kishor, J.; Worthen, S.F.; Gregory, L.J.; Aziz, Q. Exploring relationships for visceral and somatic pain with autonomic control and personality. Pain 2009, 144, 236-244. [CrossRef]

77. Perlaki, G.; Orsi, G.; Schwarcz, A.; Bodi, P.; Plozer, E.; Biczo, K.; Aradi, M.; Doczi, T.; Komoly, S.; Hejjel, L.; et al. Pain-related autonomic response is modulated by the medial prefrontal cortex: An ECG-fMRI study in men. J. Neurol. Sci. 2015, 349, 202-208. [CrossRef]

78. Petersen, K.K.; Andersen, H.H.; Tsukamoto, M.; Tracy, L.; Koenig, J.; Arendt-Nielsen, L. The effects of propranolol on heart rate variability and quantitative, mechanistic, pain profiling: A randomized placebo-controlled crossover study. Scand. J. Pain 2018, 18, 479-489. [CrossRef]

79. Picchiottino, M.; Honoré, M.; Leboeuf-Yde, C.; Gagey, O.; Cottin, F.; Hallman, D.M. The effect of a single spinal manipulation on cardiovascular autonomic activity and the relationship to pressure pain threshold: A randomized, cross-over, sham-controlled trial. Chiropr. Man. Ther. 2020, 28, 1-16. [CrossRef]

80. Piovesan, A.; Mirams, L.; Poole, H.; Moore, D.; Ogden, R. The relationship between pain-induced autonomic arousal and perceived duration. Emotion 2019, 19, 1148-1161. [CrossRef]

81. Pollatos, O.; Füstös, J.; Critchley, H.D. On the generalised embodiment of pain: How interoceptive sensitivity modulates cutaneous pain perception. Pain 2012, 153, 1680-1686. [CrossRef]

82. Pollatos, O.; Herbert, B.M.; Füstös, J.; Weimer, K.; Enck, P.; Zipfel, S. Food deprivation sensitizes pain perception. Psychophysiology 2012, 26, 1-9. [CrossRef]

83. Poulsen, C.E.; Bendixen, K.H.; Terkelsen, A.J.; May, A.; Hansen, J.; Svensson, P. Region-specific effects of trigeminal capsaicin stimulation. J. Oral Facial Pain Headache 2019, 33, 318-330. [CrossRef] [PubMed]

84. Santarcangelo, E.L.; Carli, G.; Migliorini, S.; Fontani, G.; Varanini, M.; Balocchi, R. Heart-rate control during pain and suggestions of analgesia without deliberate induction of hypnosis. Int. J. Clin. Exp. Hypn. 2008, 56, 255-269. [CrossRef] [PubMed]

85. Schneider, R. Essential oil inhaler (AromaStick ${ }^{\circledR}$ ) improves heat tolerance in the Hot Immersion Test (HIT). Results from two randomized, controlled experiments. J. Therm. Biol. 2020, 87, 102478. [CrossRef] [PubMed]

86. Sclocco, R.; Beissner, F.; Desbordes, G.; Polimeni, J.; Wald, L.; Kettner, N.W.; Kim, J.; Garcia, R.G.; Renvall, V.; Bianchi, A.M.; et al. Neuroimaging brainstem circuitry supporting cardiovagal response to pain: A combined heart rate variability/ultrahigh-field (7 T) functional magnetic resonance imaging study. Philos. Trans. R. Soc. A Math. Phys. Eng. Sci. 2016, 374, 20150189. [CrossRef]

87. Sharma, P.; Mavai, M.; Bhagat, O.L.; Murugesh, M.; Sircar, S. Slow deep breathing increases pain-tolerance and modulates cardiac autonomic nervous system. Indian J. Physiol. Pharmacol. 2017, 61, 107-113.

88. Streff, A.; Kuehl, L.K.; Michaux, G.; Anton, F. Differential physiological effects during tonic painful hand immersion tests using hot and ice water. Eur. J. Pain 2010, 14, 266-272. [CrossRef]

89. Terkelsen, A.J.; Andersen, O.K.; Molgaard, H.; Hansen, J.; Jensen, T.S. Mental stress inhibits pain perception and heart rate variability but not a nociceptive withdrawal reflex. Acta Physiol. Scand. 2004, 180, 405-414. [CrossRef]

90. Terkelsen, A.J.; Mølgaard, H.; Hansen, J.; Andersen, O.K.; Jensen, T.S. Acute pain increases heart rate: Differential mechanisms during rest and mental stress. Auton. Neurosci. 2005, 121, 101-109. [CrossRef] 
91. Terkelsen, A.J.; Bach, F.W.; Jensen, T.S. Experimental forearm immobilization in humans induces cold and mechanical hyperalgesia. J. Am. Soc. Anesthesiol. 2008, 109, 297-307. [CrossRef]

92. Tian, S.; Luo, X.; Che, X.; Xu, G. Self-compassion demonstrating a dual relationship with pain dependent on high-frequency heart rate variability. Pain Res. Manag. 2020, 2020, 3126036. [CrossRef]

93. Tousignant-Laflamme, Y.; Marchand, S. Autonomic reactivity to pain throughout the menstrual cycle in healthy women. Clin. Auton. Res. 2009, 19, 167-173. [CrossRef] [PubMed]

94. Tracy, L.M.; Jarczok, M.N.; Ellis, R.J.; Bach, C.; Hillecke, T.K.; Thayer, J.F.; Koenig, J. Heart rate variability and sensitivity to experimentally induced pain: A replication. Pain Pract. 2017, 18, 687-689. [CrossRef] [PubMed]

95. Treister, R.; Kliger, M.; Zuckerman, G.; Aryeh, I.G.; Eisenberg, E. Differentiating between heat pain intensities: The combined effect of multiple autonomic parameters. Pain 2012, 153, 1807-1814. [CrossRef] [PubMed]

96. Walter, S.; Gruss, S.; Limbrecht-Ecklundt, K.; Traue, H.C.; Werner, P.; Al-Hamadi, A.; Diniz, N.; Silva, G.M.; Andrade, A.O Automatic pain quantification using autonomic parameters. Psychol. Neurosci. 2014, 7, 363-380. [CrossRef]

97. Ye, J.J.; Lee, K.T.; Lin, J.S.; Chuang, C.C. Observing continuous change in heart rate variability and photoplethysmography-derived parameters during the process of pain production/relief with thermal stimuli. J. Pain Res. 2017, 10, 527. [CrossRef]

98. Zunhammer, M.; Eichhammer, P.; Busch, V. Do cardiorespiratory variables predict the antinociceptive effects of deep and slow breathing? Pain Med. 2013, 14, 843-854. [CrossRef]

99. Malik, M. Heart rate variability: Standards of measurement, physiological interpretation, and clinical use: Task force of the European Society of Cardiology and the North American Society for Pacing and Electrophysiology. Ann. Noninvasive Electrocardiol. 1996, 1, 151-181. [CrossRef]

100. Laborde, S.; Mosley, E.; Mertgen, A. Vagal tank theory: The three rs of cardiac vagal control functioning-resting, reactivity, and recovery. Front. Neurosci. 2018, 12, 458. [CrossRef]

101. Jester, D.J.; Rozek, E.K.; McKelley, R.A. Heart rate variability biofeedback: Implications for cognitive and psychiatric effects in older adults. Aging Ment. Health 2019, 23, 574-580. [CrossRef] 